\title{
Central extensions of the Ptolemy-Thompson group and quantized Teichmüller theory*
}

\author{
Louis Funar and Vlad Sergiescu \\ Institut Fourier BP 74, UMR 5582 \\ University of Grenoble I \\ 38402 Saint-Martin-d'Hères cedex, France \\ e-mail: \{funar, sergiesc\}@fourier.ujf-grenoble.fr
}

September 18, 2021

\begin{abstract}
The central extension of the Thompson group $T$ that arises in the quantized Teichmüller theory is 12 times the Euler class. This extension is obtained by taking a (partial) abelianization of the so-called braided Ptolemy-Thompson group introduced and studied in [23. We describe then the cyclic central extensions of $T$ by means of explicit presentations.
\end{abstract}

2000 MSC Classification: 57 M 07, 20 F 36, 20 F 38, 57 N 05.

Keywords: Thompson group, Ptolemy groupoid, infinite braid group, quantization, Teichmüller space, braided Thompson group, Euler class, discrete Godbillon-Vey class.

\section{Introduction and statements}

Fock and Goncharov ([17, 19]) improved on previous work of Faddeev, Kashaev ([15, 28]), Chekhov and Fock ([11]) and defined new families of projective unitary representations of modular groupoids associated to cluster algebras. In the particular case of $\operatorname{SL}(2, \mathbb{R})$ one obtained (projective) representations of the Ptolemy modular groupoids associated to triangulations of surfaces, arising in the quantification of the Teichmüller space. The main ingredient is the quantum dilogarithm function which permits to deform the natural action of the modular groupoid on the Teichmüller space. Thereby they will be called dilogarithmic representations. These are actually projective unitary representations, or equivalently, representations of suitable central extensions.

These representations - depending on a deformation parameter - are infinite dimensional. The general belief is that they collapse at roots of unity to finite dimensional representations which can be identified with the mapping class group representations arising from the quantum group $U_{q}(\operatorname{SL}(2, \mathbb{R}))$. Moreover, they should also coincide with the representations coming from the quantum hyperbolic invariants introduced by Baseilhac and Benedetti (see [2]).

There exists an universal setting for these constructions where the surface is the hyperbolic plane $\mathbb{H}^{2}$ (endowed with a specific triangulation, namely the Farey triangulation) and the universal Teichmüller space is the one constructed by Penner in [31. The associated modular groupoid is the Ptolemy groupoid of flips on the triangulation. As it is well-known (see [31]) there is a group structure underlying the groupoid structure that identifies the Ptolemy group of flips on the Farey triangulation to the Thompson group $T$ of piecewise$\operatorname{PSL}(2, \mathbb{Z})$ homeomorphisms of the circle (see [10]). Our aim is to identify the central extension $\widehat{T}$ of $T$ arising in the dilogarithm representations constructed in (19], section 10, [18], section 3). We refer to $\widehat{T}$ as the dilogarithmic central extension of $T$.

Following [25] the cohomology ring $H^{*}(T)$ is generated by two classes $\alpha, \chi \in H^{2}(T)$, which are called the discrete Godbillon-Vey class and respectively, the Euler class. One can obtain $\chi$ as the Euler class of the action of $T$ on the circle.

*This version: June 2009. L.F. was partially supported by the ANR Repsurf:ANR-06-BLAN-0311. This preprint is available electronically at http://www-fourier.ujf-grenoble.fr/ funar 
Moreover, central extensions of $T$ are classified up to isomorphism by their extension classes in $H^{2}(T)$. For instance the Euler class $\chi$ is the extension class of the central extension

$$
1 \rightarrow \mathbb{Z} \rightarrow \widetilde{T} \rightarrow T \rightarrow 1
$$

where $\widetilde{T} \subset \mathrm{Homeo}^{+}(\mathbb{R})$ is the group of lifts of piecewise-PSL $(2, \mathbb{Z})$ homeomorphisms of the circle to homeomorphisms of the real line $\mathbb{R}$.

Our first goal is the identification of the extension class of $\widehat{T}$. Specifically, our first main result is the following:

Theorem 1.1. The class $c_{\widehat{T}}$ of the dilogarithmic central extension $\widehat{T}$ is $c_{\widehat{T}}=12 \chi \in H^{2}(T)$.

Our approach consists of relating the dilogarithmic central extension $\widehat{T}$ to the braided Ptolemy-Thompson group $T^{*}$ introduced and studied in 23,24 . This will provide a description of $\widehat{T}$ by means of an explicit group presentation by generators and relations. The braided Ptolemy-Thompson group $T^{*}$ is an extension of $T$ by the infinite group $B_{\infty}$ of braids, which arises as a mapping class group of an infinite surface. This group is finitely presented and we are using heavily explicit presentations of related groups.

We will present a rather direct proof (using however results from [23]) showing that the dilogarithmic extension class is a multiple of the Euler class because the extension splits over the smaller Thompson group $F \subset T$. This multiple is next shown to equal 12 .

There is a general setup for studying central extensions of a finitely presented group in which all relations are given arbitrary central lifts in the extension. In the case of the group $T$ this provides a series of group presentations depending on four integer parameters $T_{n, p, q, r}$. The dilogarithmic extension $\widehat{T}$ appears in this series as $T_{1,0,0,0}$.

Although our main motivation was the result of theorem 1.1 we thought it is interesting to obtain the complete picture concerning the central extensions of $T$ in terms of explicit group presentations. This amounts to understand the cohomology 2-classes of $T$ by means of their associated extensions.

Theorem 1.2. Let $T_{n, p, q, r}$ be the group presented by the generators $\bar{\alpha}, \bar{\beta}, z$ and the relations:

$$
\begin{gathered}
(\bar{\beta} \bar{\alpha})^{5}=z^{n} \\
\bar{\alpha}^{4}=z^{p} \\
\bar{\beta}^{3}=z^{q} \\
{\left[\bar{\beta} \bar{\alpha} \bar{\beta}, \bar{\alpha}^{2} \bar{\beta} \bar{\alpha} \bar{\beta} \bar{\alpha}^{2}\right]=z^{r}} \\
{\left[\bar{\beta} \bar{\alpha} \bar{\beta}, \bar{\alpha}^{2} \bar{\beta} \bar{\alpha}^{2} \bar{\beta} \bar{\alpha} \bar{\beta} \bar{\alpha}^{2} \bar{\beta}^{2} \bar{\alpha}^{2}\right]=1} \\
{[\bar{\alpha}, z]=[\bar{\beta}, z]=1}
\end{gathered}
$$

Then each central extension of $T$ by $\mathbb{Z}$ is of the form $T_{n, p, q, r}$. Moreover, the class $c_{T_{n, p, q, r}} \in H^{2}(T)$ of the extension $T_{n, p, q, r}$ is given by:

$$
c_{T_{n, p, q, r}}=(12 n-15 p-20 q-60 r) \chi+r \alpha
$$

The extension classes behave linearly on the parameters and, in order to find their coefficients, one has to make use of several explicit (central) extensions. There are only a few such extensions in the literature, namely those coming from mapping class groups of planar surfaces with infinitely many punctures. For general central extensions we face new complications due to the presence of the discrete Godbillon-Vey class. The Ptolemy-Thompson group $T^{*}$ is one such mapping class group, but it is useless in computing the coefficients of the Godbillon-Vey class. The other extension of the same kind is the Greenberg-Sergiescu acyclic extension (see [27]) and hence the core of the proof of theorem 1.2 consists of computations within the Greenberg-Sergiescu extension. This lead us to the formula for $c_{T_{n, p, q, r, 0}}$ stated in theorem 1.2,

All over this paper the Ptolemy-Thompson group $T$ appears in every one of its instances, as a group of homeomorphisms of the circle, as the group of flips, as the group $P P S L(2, \mathbb{Z})$ and as a mapping class group. A main difficulty is to pass from one description to another one. 
The plan of the paper is as follows. We introduce in the first part the braided Ptolemy-Thompson $T^{*}$ and the induced abelianized central extension $T_{\mathrm{ab}}^{*}$. We will explain that the class $c_{T_{\mathrm{ab}}^{*}}$ is a multiple of the Euler class and provide a short proof that it should be 12 times the later. In particular we describe all central extensions associated to multiples of the Euler class. In the second part we give a quick overview of the quantization of the Teichmüller space, following mostly Fock and Goncharov's series of papers, to introduce the dilogarithmic projective representation of the Ptolemy-Thompson group. As projective representations are in one-to-one correspondence with central extensions one obtains what we will call the dilogarithmic extension $\widehat{T}$ of $T$. In the final section of this part we prove that $\widehat{T}$ and $T_{\mathrm{ab}}^{*}$ are isomorphic. The third part of the paper is devoted to the classification of all central extensions of $T$ by $\mathbb{Z}$. The major problem is to understand the behavior of the discrete Godbillon-Vey class in extensions. If the braided Ptolemy-Thompson group $T^{*}$ was the geometric model in the first part now we make use of the Greenberg-Sergiescu $\mathcal{A}_{T}$ of $T$. The method for computing the classes of extensions is inspired by Milnor's algorithm concerning the Euler class of a surface group representation. The last section contains some speculations on what should be the geometric extensions of $T$ on one hand, and the central extensions of the mapping class group of a finite (punctured) surface arising in the quantized Teichmüller theory.

Although we use some of the results from [23] and [27, quite a large amount of these papers is not essential for understanding the present paper. We also outlined the main constructions from [17] in the case of the universal Teichmüller space in order to make the paper more self-contained.

Acknowledgements. The authors are indebted to Stéphane Baseilhac, Volodya Fock, Rinat Kashaev, Christophe Kapoudjian and Greg McShane for useful discussions.

\section{Contents}

1 Introduction and statements

2 The central extension induced from the braided Ptolemy-Thompson group $T^{*}$

2.1 The Thompson groups $F$ and $T \ldots \ldots \ldots \ldots \ldots \ldots \ldots \ldots$

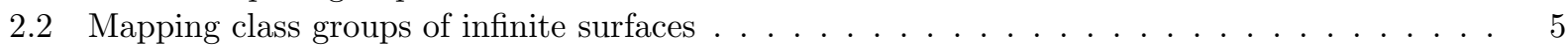

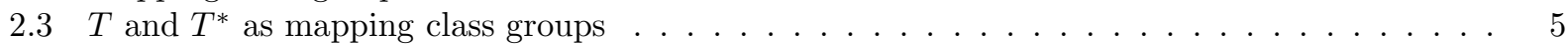

2.4 The relative abelianization of the braided Ptolemy-Thompson group $T^{*} \ldots \ldots \ldots \ldots$

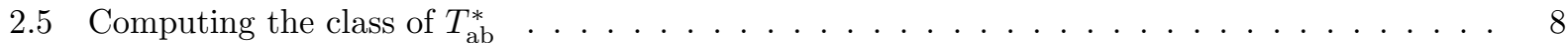

3 Quantum Teichmüller space and dilogarithmic representations $\quad 10$

3.1 The Ptolemy-Thompson group and the Ptolemy groupoid . . . . . . . . . . . . . 10

3.2 Quantum universal Teichmüller space . . . . . . . . . . . . . . . . . . . . . 11

3.3 The dilogarithmic representation of $T \ldots \ldots \ldots \ldots \ldots \ldots \ldots \ldots \ldots$

3.4 Identifying the two central extensions of $T \ldots \ldots \ldots \ldots \ldots \ldots \ldots \ldots$

4 Classification of central extensions of the group $T \quad \mathbf{1 7}$

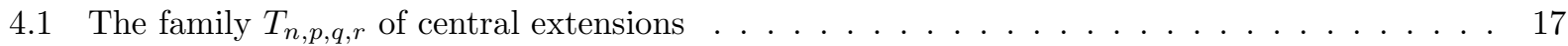

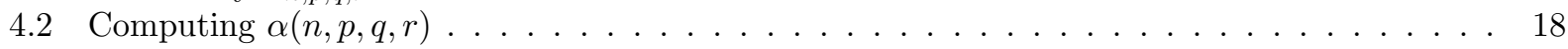

4.3 The Greenberg-Sergiescu extension $\mathcal{A}_{T} \ldots \ldots \ldots \ldots \ldots \ldots \ldots \ldots \ldots$

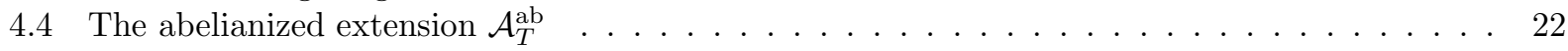

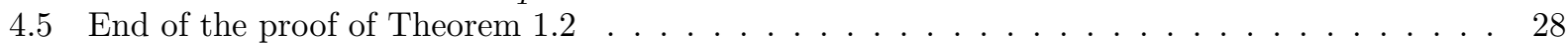

5 Odds and ends $\quad 29$

5.1 Geometric extensions . . . . . . . . . . . . . . . . . . . . . . . 29

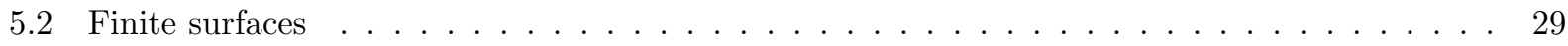




\section{The central extension induced from the braided Ptolemy-Thompson group $T^{*}$}

\subsection{The Thompson groups $F$ and $T$}

The Thompson group $F$ is the group of dyadic piecewise affine homeomorphism of $[0,1]$. We describe every element $\gamma$ of $F$ as follows. There exist two partitions of $[0,1]$ into consecutive intervals $I_{1}, I_{2}, \ldots, I_{k}$ on one side and $J_{1}, J_{2}, \ldots, J_{k}$ whose end points are dyadic numbers and $\gamma$ sends affinely each interval $I_{j}$ into its counterpart $J_{j}$, for all $j \in\{1,2, \ldots, k\}$. Thus the restriction of $\gamma$ to any interval $I_{j}$ is given by $\gamma(x)=a_{j} x+b_{j}$, where $a_{j}=2^{n_{j}}, n_{j} \in \mathbb{Z}$ and $b_{j}$ belong to the set of dyadic numbers i.e. $b_{j}=\frac{p_{j}}{2^{m_{j}}}, p_{j}, m_{j} \in \mathbb{Z}$.

The group $F$ is generated by the two elements $A$ and $B$ below:

$$
A(x)=\left\{\begin{array}{lll}
\frac{x}{2}, & \text { if } \quad x \in\left[0, \frac{1}{2}\right] \\
x-\frac{1}{4}, & \text { if } \quad x \in\left[\frac{1}{2}, \frac{3}{4}\right] \\
2 x-1, & \text { if } \quad x \in\left[\frac{3}{4}, 1\right]
\end{array}, B(x)=\left\{\begin{array}{lll}
x, & \text { if } & x \in\left[0, \frac{1}{2}\right] \\
\frac{x}{2}+\frac{1}{4}, & \text { if } & x \in\left[\frac{1}{2}, \frac{3}{4}\right] \\
x-\frac{1}{8}, & \text { if } & x \in\left[\frac{3}{4}, \frac{7}{8}\right] \\
2 x-1, & \text { if } & x \in\left[\frac{7}{8}, 1\right]
\end{array}\right.\right.
$$

Moreover, the group $F$ has the presentation

$$
F=\left\langle A, B ;\left[A B^{-1}, A^{-1} B A\right]=1,\left[A B^{-1}, A^{-2} B A^{2}\right]=1\right\rangle
$$

There is a geometric encoding of elements of $F$ as pairs of (stable) finite rooted binary trees. Each finite rooted binary tree encodes a subdivision of $[0,1]$ into dyadic intervals. Adding two descending edges to a vertex amounts to subdivide the respective interval into two equal halves. Given the two subdivisions the element of $F$ sending one into the other is uniquely determined. The pair of trees is determined up to stabilization, namely adding extra couples of descending edges to corresponding vertices in both trees.
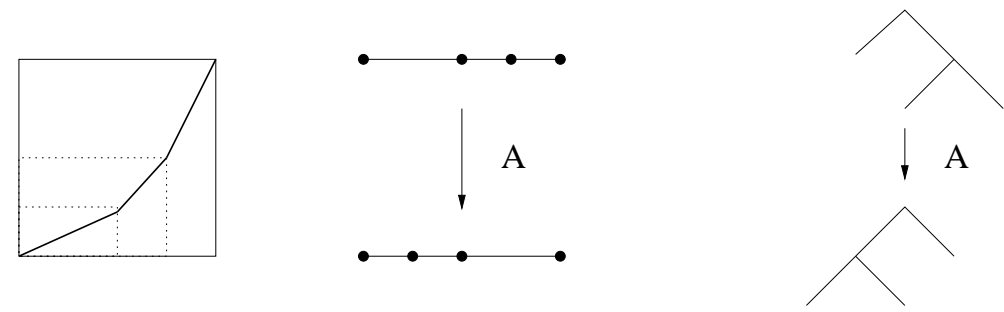

A
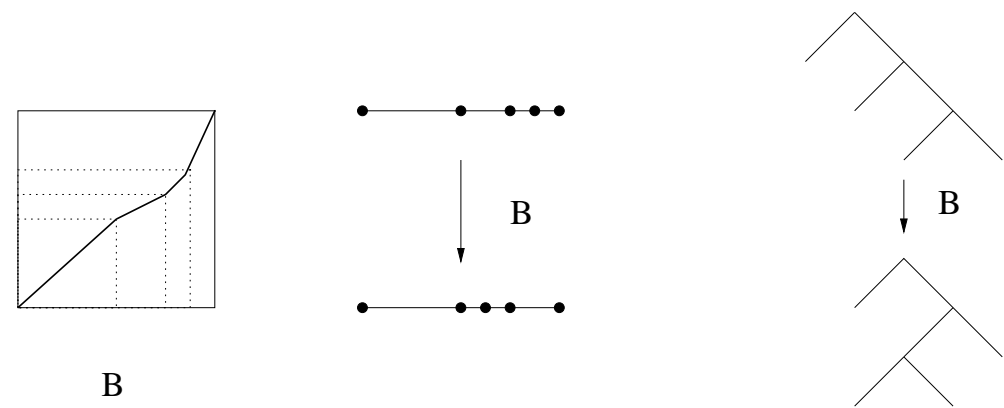

B

The Thompson group $T$ is the group of dyadic piecewise affine homeomorphisms of $S^{1}=[0,1] / 0 \sim 1$. It is not hard to see that $A, B$ and $C$ generate $T$, where

$$
C(x)=\left\{\begin{array}{lll}
\frac{x}{2}+\frac{3}{4}, & \text { if } & x \in\left[0, \frac{1}{2}\right] \\
2 x-1, & \text { if } & x \in\left[\frac{1}{2}, \frac{3}{4}\right] \\
x-\frac{1}{4}, & \text { if } & x \in\left[\frac{3}{4}, 1\right]
\end{array}\right.
$$

The presentation of $T$ in terms of the generators $A, B, C$ consists of the two relations above with four more relations to be added:

$$
T=\left\langle\begin{array}{ll}
A, B, C ; & C A=\left(A^{-1} C B\right)^{2},\left(A^{-1} C B\right)\left(A^{-1} B A\right)=B\left(A^{-2} C B^{2}\right), \\
& C^{3}=1, C=B A^{-1} C B,\left[A B^{-1}, A^{-1} B A\right]=1, \quad\left[A B^{-1}, A^{-2} B A^{2}\right]=1
\end{array}\right\rangle
$$


We can associate a pair of (stable) trees to encode an element of $T$, as well, but we have to specify additionally, where the origin is sent to. We denote the image of the origin in the trees by marking the leftmost leaf of its domain. Usually the origin is fixed to be the leftmost leaf in the first tree. For instance, the element $C$ has the following description:

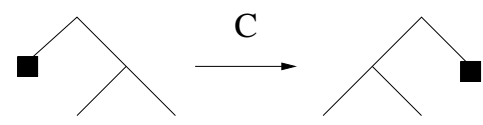

Remark 2.1. The group $T$ has also another presentation with generators $\alpha$ and $\beta$ and relations

$$
\begin{gathered}
\alpha^{4}=\beta^{3}=1 \\
{\left[\beta \alpha \beta, \alpha^{2} \beta \alpha \beta \alpha^{2}\right]=1} \\
{\left[\beta \alpha \beta, \alpha^{2} \beta^{2} \alpha^{2} \beta \alpha \beta \alpha^{2} \beta \alpha^{2}\right]=1} \\
(\beta \alpha)^{5}=1
\end{gathered}
$$

If we set $A=\beta \alpha^{2}, B=\beta^{2} \alpha$ and $C=\beta^{2}$ then we obtain the generators $A, B, C$ of the group $T$, considered above. Then the two commutativity relations above are equivalent to

$$
\left[A B^{-1}, A^{-1} B A\right]=1, \quad\left[A B^{-1}, A^{-2} B A^{2}\right]=1
$$

\subsection{Mapping class groups of infinite surfaces}

Definition 2.1. A rigid structure $d$ on the surface $\Sigma$ is a decomposition of $\Sigma$ into 2-disks with disjoint interiors, called elementary pieces. We suppose that the closures of the elementary pieces are still 2-disks.

We assume that we are given a family $F$, called the family of admissible sub-surfaces of $\Sigma$, of compact sub-surfaces of $\Sigma$ such that each member of $F$ is a finite union of elementary pieces.

Given the data $(\Sigma, d, F)$ we can associate the asymptotic mapping class group $\mathcal{M}(\Sigma, d, F)$ as follows. We restrict first to those homeomorphisms that act in the simplest possible way at infinity.

Definition 2.2. A homeomorphism $\varphi$ between two surfaces endowed with rigid structures is rigid if it sends the rigid structure of one surface onto the rigid structure of the other.

The homeomorphism $\varphi: \Sigma \rightarrow \Sigma$ is said to be asymptotically rigid if there exists some admissible subsurface $C \subset \Sigma$, called a support for $\varphi$, such that $\varphi(C) \subset \Sigma$ is also an admissible subsurface of $\Sigma$ and the restriction $\left.\varphi\right|_{\Sigma-C}: \Sigma-C \rightarrow \Sigma-\varphi(C)$ is rigid.

As it is customary when studying mapping class groups we consider now isotopy classes of such homeomorphisms.

Definition 2.3. The group $\mathcal{M}(\Sigma, d, F)$ of isotopy classes of asymptotically rigid homeomorphisms is called the asymptotic mapping class group of $\Sigma$ corresponding to the rigid structure $d$ and family of admissible sub-surfaces $F$.

Remark 2.2. Two asymptotically rigid homeomorphisms that are isotopic should be isotopic among asymptotically rigid homeomorphisms.

\section{$2.3 \quad T$ and $T^{*}$ as mapping class groups}

The surfaces below will be oriented and all homeomorphisms considered in the sequel will be orientationpreserving, unless the opposite is explicitly stated. Actions in the sequel are left actions and the composition of maps is the usual one, namely we start composing from right to the left.

Definition 2.4. The ribbon tree $D$ is the planar surface obtained by thickening in the plane the infinite binary tree. We denote by $D^{*}$ the ribbon tree with infinitely many punctures, one puncture for each edge of the tree. A homeomorphism of $D^{*}$ is a homeomorphism of $D$ which permutes the punctures $D^{*}$. 
Definition 2.5. A rigid structure on $D$ or $D^{*}$ is a decomposition into hexagons by means of a family of arcs whose endpoints are on the boundary of D. Each hexagon contains no puncture within its interior but each arc passes through a unique puncture in the case of $D^{*}$. It is assumed that these arcs are pairwise non-homotopic in D, by homotopies keeping the boundary points of the arcs on the boundary of D. The choice of a rigid structure of reference is called the canonical rigid structure. The canonical rigid structure of the ribbon tree $D$ is such that each arc of this rigid structure crosses once and transversely a unique edge of the tree. The canonical rigid structure on $D^{*}$ is assumed to coincide with the canonical rigid structure of $D$ when forgetting the punctures. See the Figure below:

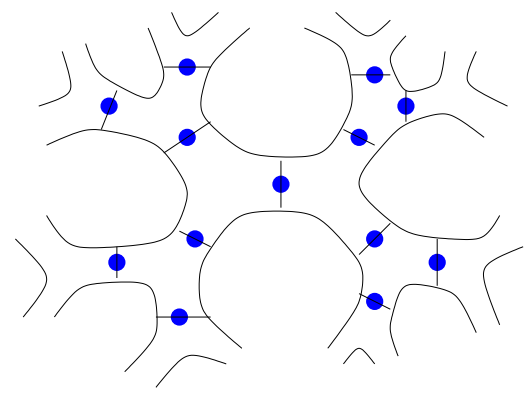

A planar subsurface of $D$ or $D^{*}$ is admissible if it is a connected finite union of hexagons coming from the canonical rigid structure.

One denotes by $T$ and $T^{*}$ the group of isotopy classes of asymptotically rigid homeomorphisms of $D$ and $D^{*}$, respectively.

Remark 2.3. There exists a cyclic order on the frontier arcs of an admissible subsurface induced by the planarity. An asymptotically rigid homeomorphism necessarily preserves the cyclic order of the frontier for any admissible subsurface.

The mapping class group $T$ is isomorphic to the Thompson group which is commonly denoted $T$. This fact has been widely developed in [29] and [23. We consider the following elements of $T$, defined as mapping classes of asymptotically rigid homeomorphisms:

- The support of the element $\beta$ is the central hexagon on the figure below. Further, $\beta$ acts as the counterclockwise rotation of order three which permutes the three branches of the ribbon tree issued from the hexagon.

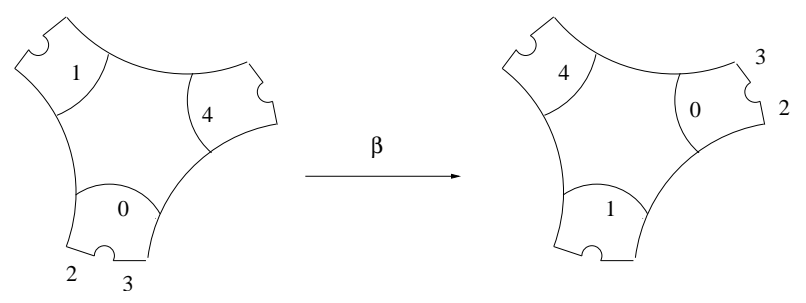

In fact, $\beta$ is globally rigid.

- The support of $\alpha$ is the union of two adjacent hexagons, one of them being the support of $\beta$. Then $\alpha$ rotates counterclockwise the support of angle $\frac{\pi}{2}$, by permuting the four branches of the ribbon tree issued from the support.

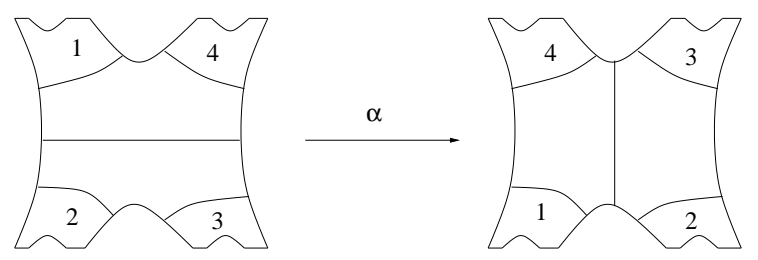

Note that $\alpha$ is not globally rigid, but $\alpha^{2}$ is. 


\subsection{The relative abelianization of the braided Ptolemy-Thompson group $T^{*}$}

Recall from 23, 24, that there exists a natural surjection homomorphism $T^{*} \rightarrow T$ between the two mapping class groups, which is obtained by forgetting the punctures. Its kernel is the infinite braid group $B_{\infty}$ consisting of those braids in the punctures of $D^{*}$ that move non-trivially only finitely many punctures. In other words $B_{\infty}$ is the direct limit of an ascending sequence of braid groups associated to an exhaustion of $D^{*}$ by punctured disks. This yields the following exact sequence description of $T^{*}$ :

$$
1 \rightarrow B_{\infty} \rightarrow T^{*} \rightarrow T \rightarrow 1
$$

Observe that $H_{1}\left(B_{\infty}\right)=\mathbb{Z}$. Thus, the abelianization homomorphism $B_{\infty} \rightarrow H_{1}\left(B_{\infty}\right)=\mathbb{Z}$ induces a central extension $T_{\mathrm{ab}}^{*}$ of $T$, where one replaces $B_{\infty}$ by its abelianization $H_{1}\left(B_{\infty}\right)$, as in the diagram below:

$$
\begin{aligned}
& 1 \rightarrow B_{\infty} \rightarrow T^{*} \rightarrow T \rightarrow 1
\end{aligned}
$$

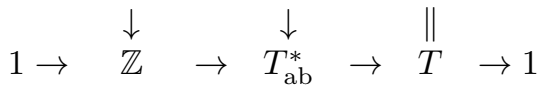

Then $T_{\mathrm{ab}}^{*}$ is the relative abelianization of $T^{*}$ over $T$. We are not only able to make computations in the mapping class group $T^{*}$ and thus in $T_{\mathrm{ab}}^{*}$, but also to interpret the algebraic relations in $T_{\mathrm{ab}}^{*}$ in geometric terms.

Proposition 2.1. The group $T_{\mathrm{ab}}^{*}$ has the presentation with three generators $\alpha_{\mathrm{ab}}^{*}, \beta_{\mathrm{ab}}^{*}$ and $z$ and the relations

$$
\begin{gathered}
\alpha_{\mathrm{ab}}^{*}{ }^{4}=\beta_{\mathrm{ab}}^{*}{ }^{3}=1,\left(\beta_{\mathrm{ab}}^{*} \alpha_{\mathrm{ab}}^{*}\right)^{5}=z,\left[\alpha_{\mathrm{ab}}^{*}, z\right]=1,\left[\beta_{\mathrm{ab}}^{*}, z\right]=1 \\
{\left[\beta_{\mathrm{ab}}^{*} \alpha_{\mathrm{ab}}^{*} \beta_{\mathrm{ab}}^{*}, \alpha_{\mathrm{ab}}^{*}{ }^{2} \beta_{\mathrm{ab}}^{*} \alpha_{\mathrm{ab}}^{*} \beta_{\mathrm{ab}}^{*} \alpha_{\mathrm{ab}}^{*}{ }^{2}\right]=\left[\beta_{\mathrm{ab}}^{*} \alpha_{\mathrm{ab}}^{*} \beta_{\mathrm{ab}}^{*}, \alpha_{\mathrm{ab}}^{*}{ }^{2} \beta_{\mathrm{ab}}{ }^{2} \alpha_{\mathrm{ab}}^{*}{ }^{2} \beta_{\mathrm{ab}}^{*} \alpha_{\mathrm{ab}}^{*} \beta_{\mathrm{ab}}^{*} \alpha_{\mathrm{ab}}^{*}{ }^{2} \beta_{\mathrm{ab}}^{*} \alpha_{\mathrm{ab}}^{*}{ }^{2}\right]=1}
\end{gathered}
$$

Moreover the projection map $T_{\mathrm{ab}}^{*} \rightarrow T$ sends $\alpha_{\mathrm{ab}}^{*}$ to $\alpha, \beta_{\mathrm{ab}}^{*}$ to $\beta$ and $z$ to identity.

Proof. Recall from 23 that $T^{*}$ is generated by two elements $\alpha^{*}$ and $\beta^{*}$ below.

- The support of the element $\beta^{*}$ of $T^{*}$ is the central hexagon. Further $\beta^{*}$ acts as the counterclockwise rotation of order three which permutes cyclically the punctures. One has $\beta^{* 3}=1$.

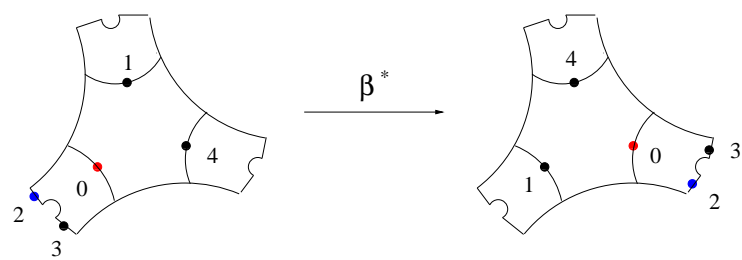

- The support of the element $\alpha^{*}$ of $T^{*}$ is the union of two adjacent hexagons, one of them being the support of $\beta^{*}$. Then $\alpha^{*}$ rotates counterclockwise the support of angle $\frac{\pi}{2}$, by keeping fixed the central puncture. One has $\alpha^{* 4}=1$.

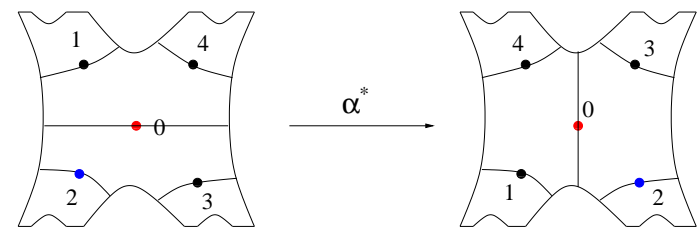

Let now $e$ be a simple arc in $D^{*}$ which connects two punctures. We associate a braiding $\sigma_{e} \in B_{\infty}$ to $e$ by considering the homeomorphism that moves clockwise the punctures at the endpoints of the edge $e$ in a small neighborhood of the edge, in order to interchange their positions. This means that, if $\gamma$ is an arc transverse to $e$, then the braiding $\sigma_{e}$ moves $\gamma$ on the left when it approaches $e$. Such a braiding will be called positive, while $\sigma_{e}^{-1}$ is negative. 


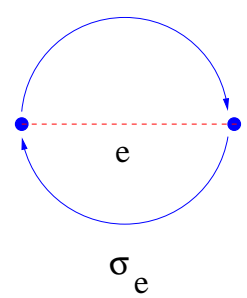

It is known that $B_{\infty}$ is generated by the braids $\sigma_{e}$ where $e$ runs over the edges of the binary tree with vertices at punctures. Let $\iota: B_{\infty} \rightarrow T^{*}$ be the inclusion. It is proved in [23] that the braid generator $\sigma_{[02]}$ associated to the edge joining the punctures numbered 0 and 2 has the image

$$
\iota\left(\sigma_{[02]}\right)=\left(\beta^{*} \alpha^{*}\right)^{5}
$$

because we have
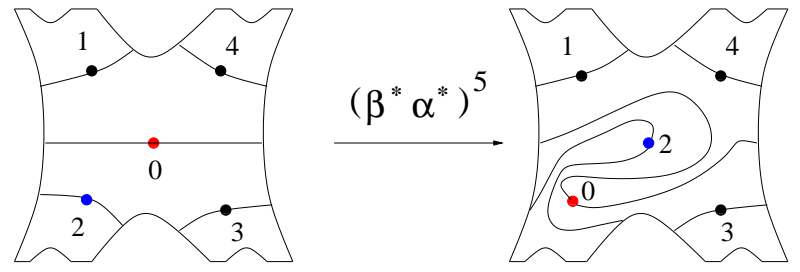

Recall next that all braid generators $\sigma_{e}$ are conjugate and call $z$ their image in $T_{\mathrm{ab}}^{*}$. It follows that $T_{\mathrm{ab}}^{*}$ is an extension of $T$ by $\mathbb{Z}$. Moreover, it is simple to check that $\alpha^{*} \sigma_{[02]} \alpha^{-1}$ is also a braid generator, namely $\sigma_{\left[\alpha^{*}(0) \alpha^{*}(2)\right]}$. The same holds true for $\beta^{*} \sigma_{[02]} \beta^{*-1}=\sigma_{\left[\beta^{*}(0) \beta^{*}(2)\right]}$. This implies that the extension $T_{\mathrm{ab}}^{*}$ is central.

In particular, a presentation of $T_{\mathrm{ab}}^{*}$ can be obtained by looking at the lifts of relations in $T$, together with those coming from the fact that $z$ is central.

The first set relations above are obviously satisfied by $T_{\mathrm{ab}}^{*}$. Finally recall from [23] that $T^{*}$ splits over the smaller Thompson group $F$ and thus the following relations hold true in $T^{*}$ :

$$
\left[\beta^{*} \alpha^{*} \beta^{*}, \alpha^{* 2} \beta^{*} \alpha^{*} \beta^{*} \alpha^{* 2}\right]=\left[\beta^{*} \alpha^{*} \beta^{*}, \alpha^{* 2} \beta^{* 2} \alpha^{* 2} \beta^{*} \alpha^{*} \beta^{*} \alpha^{* 2} \beta^{*} \alpha^{* 2}\right]=1
$$

Thus the second set of relations are automatically verified in $T_{\mathrm{ab}}^{*}$. Since these relations form a complete set of lifts of relations presenting $T$ and $z$ is central, then they represent a complete system of relations in $T_{\mathrm{ab}}^{*}$. This ends the proof.

\subsection{Computing the class of $T_{\mathrm{ab}}^{*}$}

Lemma 2.1. The class $c_{T_{\mathrm{ab}}^{*}}$ is a multiple of the Euler class.

Proof. Since $T^{*}$ splits over Thompson group $F \subset T$ (see [23]) it follows that $T_{\text {ab }}^{*}$ also splits over $F$. Therefore the extension class $c_{T_{\mathrm{ab}}^{*}}$ lies in the kernel of the restriction map $H^{2}(T) \rightarrow H^{2}(F)$. According to [25] the kernel is generated by the Euler class.

Let us introduce the group $T_{n, p, q}$ presented by the generators $\bar{\alpha}, \bar{\beta}, z$ and the relations:

$$
\begin{gathered}
(\bar{\beta} \bar{\alpha})^{5}=z^{n} \\
\bar{\alpha}^{4}=z^{p} \\
\bar{\beta}^{3}=z^{q} \\
{\left[\bar{\beta} \bar{\alpha} \bar{\beta}, \bar{\alpha}^{2} \bar{\beta} \bar{\alpha} \bar{\beta} \bar{\alpha}^{2}\right]=1} \\
{\left[\bar{\beta} \bar{\alpha} \bar{\beta}, \bar{\alpha}^{2} \bar{\beta} \bar{\alpha}^{2} \bar{\beta} \bar{\alpha} \bar{\beta} \bar{\alpha}^{2} \bar{\beta}^{2} \bar{\alpha}^{2}\right]=1} \\
{[\bar{\alpha}, z]=[\bar{\beta}, z]=1}
\end{gathered}
$$


Recall from proposition 2.1 that $T_{\mathrm{ab}}^{*}=T_{1,0,0}$. It is easy to see that $T_{n, p, q}$ are central extensions of $T$ by $\mathbb{Z}$. Because of the last two commutation relations the extension $T_{n, p, q}$ splits over the Thompson group $F$. Thus the restriction of $c_{T_{n, p, q}}$ to $F$ vanishes and a fortiori the restriction to the commutator subgroup $F^{\prime} \subset F$. According to 25] we have $H^{2}\left(F^{\prime}\right)=\mathbb{Z} \alpha$ where $\alpha$ is the discrete Godbillon-Vey class. Thus the map $H^{2}(T) \rightarrow H^{2}\left(F^{\prime}\right)$ is the projection $\mathbb{Z} \alpha \oplus \mathbb{Z} \chi \rightarrow \mathbb{Z} \alpha$. Since $c_{\widehat{T}}$ belongs to the kernel of $H^{2}(T) \rightarrow H^{2}\left(F^{\prime}\right)$. This proves that $c_{T_{n, p, q}} \in \mathbb{Z} \chi$. Set $c_{T_{n, p, q}}=\chi(n, p, q) \chi$.

Proposition 2.2. We have $\chi(n, p, q)=12 n-15 p-20 q$.

Proof. Observe first that:

Lemma 2.2. The function $\chi(n, p, q): \mathbb{Z}^{3} \rightarrow \mathbb{Z}$ is linear.

Proof. The group $T$ contains all finite cyclic subgroups. Let us fix some positive integer $k$ and set $y_{k} \in T$ for an element of order $k$. Denote by $G_{k} \subset T$ the cyclic subgroup generated by $y_{k}$.

We want to compute the restriction $\left.c_{T_{n, p, q}}\right|_{G_{k}}$, namely the image of $c_{T_{n, p, q}}$ under the obvious morphism $H^{2}(T) \rightarrow H^{2}\left(G_{k}\right)$. Since $G_{k}$ is cyclic $H^{2}\left(G_{k}\right)$ is the cyclic $\mathbb{Z} / k \mathbb{Z}$ of order $k$, generated by the restriction of the Euler class $\left.\chi\right|_{G_{k}}$. In particular,

$$
\left.c_{T_{n, p, q}}\right|_{G_{k}}=\left.\chi(n, p, q) \chi\right|_{G_{k}} \in \mathbb{Z} / k \mathbb{Z}=H^{2}\left(G_{k}\right)
$$

On the other hand we can compute the Euler class of a central extension of a cyclic subgroup of $T \subset$ Homeo $^{+}\left(S^{1}\right)$ by means of the following Milnor-Wood algorithm. Assume that $y_{k}=w_{k}(\alpha, \beta)$ is given by a word in the generators $\alpha, \beta$ of $T$. Consider next the word $\overline{y_{k}}=w_{k}(\bar{\alpha}, \bar{\beta}) \in T_{n, p, q}$. Then ${\overline{y_{k}}}^{k}=z^{e}$ for some $e$ and the Euler class is given by the value of $e$ modulo $k$.

However $\bar{y}_{k}^{k}$ is an element of the center of $T_{n, p, q}$ and thus it is a product of conjugates of $(\bar{\beta} \bar{\alpha})^{5}, \bar{\alpha}^{4}, \bar{\beta}^{3}$ and the commutation relations (which are not involving $z$ ). If $\varphi_{n, p, q}(w)$ is the additive map that associates to any sub-word of $w$ from the subset $\left\{(\bar{\beta} \bar{\alpha})^{5}, \bar{\alpha}^{4}, \bar{\beta}^{3}\right\}$ respectively $n, p$ and $q$ it follows that

$$
\varphi_{n, p, q}(w)=n \varphi_{1,0,0}(w)+p \varphi_{0,1,0}(w)+q \varphi_{0,0,1}(w)
$$

In particular, by choosing $w=w_{k}(\bar{\alpha}, \bar{\beta})^{k}$ we obtain that the coefficient of the Euler class $\chi(n, p, q, r)$ verifies

$$
\chi(n, p, q)=n \chi(1,0,0)+p \chi(0,1,0)+q \chi(0,0,1)(\bmod k)
$$

This equality holds for all natural $k$ and therefore $\chi(n, p, q)$ is linear.

End of the proof of the proposition 2.2. By the definition of the extension class $\chi(n, p, q)$ takes the same value for those $T_{n, p, q}$ that are isomorphic by an isomorphism inducing identity on $T$ and on the center. Such isomorphisms $L$ have the form $L(\bar{\alpha})=\bar{\alpha} z^{x}$ and $L(\bar{\beta})=\bar{\beta} z^{y}$. Thus $T_{n, p, q}$ is isomorphic as extension to $T_{n^{\prime}, p^{\prime}, q^{\prime}}$ if and only if there exists integers $x, y \in Z$ such that

$$
n=n^{\prime}+5 x+5 y, p=p^{\prime}+4 x, q=q^{\prime}+3 y
$$

In particular the linear form $\chi(n, p, q)$ should be invariant by the transforms corresponding to arbitrary $x, y \in \mathbb{Z}$ and thus it should be a multiple of $12 n-15 p-20 q$.

Last, the extension $\widetilde{T} \subset \operatorname{Homeo}^{+}(\mathbb{R})$ is known to have Euler number 1 . The central element $z$ acts as the unit translation on the line and in order to identify it with an element of the family $T_{n, p, q}$ it suffices to compute the rotation index associated to the elements $\alpha^{4}, \beta^{3}$ and $(\beta \alpha)^{5}$. One obtains that $\widetilde{T}$ is actually isomorphic to $T_{3,1,1}$. Thus $\chi(n, p, q)$ is precisely given by the claimed linear form.

Corollary 2.1. We have $c_{T_{\mathrm{ab}}^{*}}=12 \chi$.

Remark 2.4. The extension $T^{*} \rightarrow T$ splits also over the subgroup $\left\langle\alpha^{2}, \beta\right\rangle$ which is isomorphic to $P S L(2, \mathbb{Z})$ (see [23]). This implies that $c_{T_{\mathrm{ab}}^{*}}$ is a multiple of $6 \chi$. 


\section{Quantum Teichmüller space and dilogarithmic representations}

\subsection{The Ptolemy-Thompson group and the Ptolemy groupoid}

We will use the terms triangulation of $\mathbb{H}^{2}$ for ideal locally finite triangulations of the hyperbolic space $\mathbb{H}^{2}$ i.e. a countable locally finite collection of geodesics whose complementary regions are triangles. The vertices of the triangulation are the asymptotes of the constitutive geodesics, viewed as points of the circle at infinity $S_{\infty}^{1}$.

Consider the basic ideal triangle having vertices at $1,-1, \sqrt{-1} \in S_{\infty}^{1}$ in the unit disk model $\mathbb{D}$ of $\mathbb{H}^{2}$. The orbits of its sides by the group $P S L(2, \mathbb{Z})$ is the so-called Farey triangulation $\tau_{0}$.

A triangulation $\tau$ is marked if one fixes a distinguished oriented edge (abbreviated d.o.e.) $\vec{a}$ of it. The standard marking of the Farey triangulation $\tau_{0}$ is the oriented edge $\overrightarrow{a_{0}}$ joining -1 to 1 .

We define next a marked tessellation of $\mathbb{H}^{2}$ to be an equivalence class of marked triangulations of $\mathbb{H}^{2}$ with respect to the action of $\operatorname{PSL}(2, \mathbb{R})$. Since the action of $\operatorname{PSL}(2, \mathbb{R})$ is 3 -transitive each tessellation can be uniquely represented by its canonical marked triangulation containing the basic ideal triangle and whose d.o.e. is $\overrightarrow{a_{0}}$. The marked tessellation is of Farey-type if its canonical marked triangulation has the same vertices and all but finitely many triangles (or sides) as the Farey triangulation. Unless explicitly stated otherwise all tessellations considered in the sequel will be Farey-type tessellations. In particular, the ideal triangulations have the same vertices as $\tau_{0}$ and coincide with $\tau_{0}$ for all but finitely many ideal triangles.

Definition 3.1. The objects of the (universal) Ptolemy groupoid Pt are the marked tessellations of Fareytype. The morphisms between two objects $\left(\tau_{1}, \overrightarrow{a_{1}}\right)$ and $\left(\tau_{2}, \overrightarrow{a_{2}}\right)$ are eventually trivial permutations maps $\phi$ : $\tau_{1} \rightarrow \tau_{2}$ such that $\phi\left(\overrightarrow{a_{1}}\right)=\overrightarrow{a_{2}}$. When marked tessellations are represented by their canonical triangulations, the later coincide for all but finitely many triangles. Recall that $\phi$ is said to be eventually trivial if the induced correspondence at the level of canonical triangulations is the identity for all but finitely many edges.

We define now particular elements of $P t$ called flips. Let $e$ be an edge of the marked tessellation represented by the canonical marked triangulation $(\tau, \vec{a})$. The result of the flip $F_{e}$ on $\tau$ is the triangulation $F_{e}(\tau)$ obtained from $\tau$ by changing only the two neighboring triangles containing the edge $e$, according to the picture below:

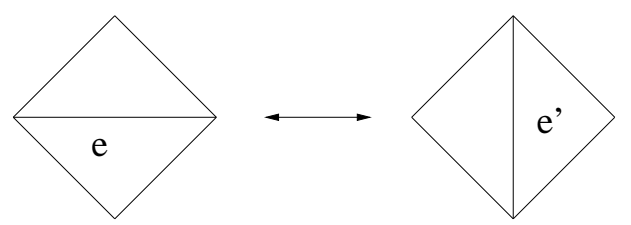

This means that we remove $e$ from $\tau$ and further add the new edge $e^{\prime}$ in order to get $F_{e}(\tau)$. In particular there is a natural correspondence $\phi: \tau \rightarrow F_{e}(\tau)$ sending $e$ to $e^{\prime}$ and identity for all other edges. The result of a flip is the new triangulation together with this edges' correspondence.

If $e$ is not the d.o.e. of $\tau$ then $F_{e}(\vec{a})=\vec{a}$. If $e$ is the d.o.e. of $\tau$ then $F_{e}(\vec{a})=\overrightarrow{e^{\prime}}$, where the orientation of $\overrightarrow{e^{\prime}}$ is chosen so that the frame $\left(\vec{e}, \overrightarrow{e^{\prime}}\right)$ is positively oriented.

We define now the flipped tessellation $F_{e}((\tau, \vec{a}))$ to be the tessellation $\left(F_{e}(\tau), F_{e}(\vec{a})\right)$. It is proved in [31] that flips generate the Ptolemy groupoid i.e. any element of $P t$ is a composition of flips.

For any marked tessellation $(\tau, \vec{a})$ there is defined a characteristic map $Q_{\tau}: \mathbb{Q}-\{-1,1\} \rightarrow \tau$. Assume that $\tau$ is the canonical triangulation representing this tessellation. We label first by $\mathbb{Q} \cup \infty$ the vertices of $\tau$, by induction:

1. -1 is labeled by $0 / 1,1$ is labeled by $\infty=1 / 0$ and $\sqrt{-1}$ is labeled by $-1 / 1$.

2. If we have a triangle in $\tau$ having two vertices already labeled by $a / b$ and $c / d$ then its third vertex is labeled $(a+c) /(b+d)$. Notice that vertices in the upper half-plane are labeled by negative rationals and those from the lower half-plane by positive rationals.

As it is well-known this labeling produces a bijection between the set of vertices of $\tau$ and $\mathbb{Q} \cup \infty$. 
Let now $e$ be an edge of $\tau$, which is different from $\vec{a}$. Let $v(e)$ be the vertex opposite to $e$ of the triangle $\Delta$ of $\tau$ containing $e$ in its frontier and lying in the component of $\mathbb{D}-e$ which does not contain $\vec{a}$. We associate then to $e$ the label of $v(e)$. Give also $\vec{a}$ the label $0 \in \mathbb{Q}$. This way one obtains a bijection $Q_{\tau}: \mathbb{Q}-\{-1,1\} \rightarrow \tau$.

Remark that if $\left(\tau_{1}, \overrightarrow{a_{1}}\right)$ and $\left(\tau_{2}, \overrightarrow{a_{2}}\right)$ are marked tessellations then there exists a unique map $f$ between their vertices sending triangles to triangles and marking on the marking. Then $f \circ Q_{\tau_{1}}=Q_{\tau_{2}}$.

The role played by $Q_{\tau}$ is to allow flips to be indexed by the rationals and not on edges of $\tau$.

Definition 3.2. Let FT be the set of marked tessellations of Farey-type. Define the action of the free monoid $M$ generated by $\mathbb{Q}-\{-1,1\}$ on $\mathrm{FT}$ by means of:

$$
q \cdot(\tau, \vec{a})=F_{Q_{\tau}(q)}(\tau, \vec{a}), \text { for } q \in \mathbb{Q}-\{-1,1\},(\tau, \vec{a}) \in \mathrm{FT}
$$

We set $f \sim f^{\prime}$ on $M$ if the two actions of $f$ and $f^{\prime}$ on FT coincide. Then the induced composition law on $M / \sim$ is a monoid structure for which each element has an inverse. This makes $M / \sim$ a group, which is called the Ptolemy group $T$ (see [31] for more details).

In particular it makes sense to speak of flips in the present case and thus flips generate the Ptolemy group.

The notation $T$ for the Ptolemy group is not misleading because this group is isomorphic to the Thompson group $T$ and for this reason, we preferred to call it the Ptolemy-Thompson group.

Given two marked tessellations $\left(\tau_{1}, \overrightarrow{a_{1}}\right)$ and $\left(\tau_{2}, \overrightarrow{a_{2}}\right)$ the combinatorial isomorphism $f: \tau_{1} \rightarrow \tau_{2}$ from above provides a map between the vertices of the triangulations, which are identified with $P^{1}(\mathbb{Q}) \subset S_{\infty}^{1}$. This map extends continuously to a homeomorphism of $S_{\infty}^{1}$, which is piecewise- $P S L(2, \mathbb{Z})$. This establishes an isomorphism between the Ptolemy group and the group of piecewise- $P S L(2, \mathbb{Z})$ homeomorphisms of the circle.

An explicit isomorphism with the group $T$ in the form introduced above was provided by Lochak and Schneps (see [30]). Send $\alpha$ to the flip $F_{a}$ of $\left(\tau_{0}, \overrightarrow{a_{0}}\right)$ and $\beta$ to the element $\left(\left(\tau_{0}, \overrightarrow{a_{0}}\right),\left(\tau_{0}, \overrightarrow{a_{1}}\right)\right)$ of the Ptolemy group, where $\overrightarrow{a_{1}}$ is the oriented edge in the basic triangle of the Farey triangulation $\tau_{0}$ next to $\overrightarrow{a_{0}}$.

\subsection{Quantum universal Teichmüller space}

Here and henceforth, for the sake of brevity, we will use the term tessellation instead of marked tessellation. For each tessellation $\tau$ let $E(\tau)$ be the set of its edges. We associate further a skew-symmetric matrix $\varepsilon(\tau)$ with entries $\varepsilon_{e f}$, for all $e, f \in E(\tau)$, as follows. If $e$ and $f$ do not belong to the same triangle of $\tau$ or $e=f$ then $\varepsilon_{e f}=0$. Otherwise, $e$ and $f$ are distinct edges belonging to the same triangle of $\tau$ and thus have a common vertex. We obtain $f$ by rotating $e$ in the plane along that vertex such that the moving edge is sweeping out the respective triangle of $\tau$. If we rotate clockwisely then $\varepsilon_{e f}=+1$ and otherwise $\varepsilon_{e f}=-1$.

The couple $(E(\tau), \varepsilon(\tau))$ is called a seed in [19. Observe, that in this particular case seeds are completely determined by tessellations.

Let $\left(\tau, \tau^{\prime}\right)$ be a flip $F_{e}$ in the edge $e \in E(\tau)$. Then the associated seeds $(E(\tau), \varepsilon(\tau))$ and $\left(E\left(\tau^{\prime}\right), \varepsilon\left(\tau^{\prime}\right)\right)$ are obtained one from the other by a mutation in the direction $e$. Specifically, this means that there is an isomorphism $\mu_{e}: E(\tau) \rightarrow E\left(\tau^{\prime}\right)$ such that

$$
\varepsilon\left(\tau^{\prime}\right)_{\mu_{e}(s) \mu_{e}(t)}= \begin{cases}-\varepsilon_{s t}, & \text { if } e=s \text { or } e=t, \\ \varepsilon_{s t}, & \text { if } \varepsilon_{s e} \varepsilon_{e t} \leq 0, \\ \varepsilon_{s t}+\left|\varepsilon_{s e}\right| \varepsilon_{e t}, & \text { if } \varepsilon_{s e} \varepsilon_{e t}>0\end{cases}
$$

The map $\mu_{e}$ comes from the natural identification of the edges of the two respective tessellations out of $e$ and $F_{e}(e)$.

This algebraic setting appears in the description of the universal Teichmüller space $\mathcal{T}$. Its formal definition (see [17, 18]) is the set of positive real points of the cluster $\mathcal{X}$-space related to the set of seeds above. However, we can give a more intuitive description of it, following [31. Specifically, $\mathcal{T}$ is the space of all marked tessellations (denoted $\mathcal{T}$ ess in [31]). Each tessellation $\tau$ gives rise to a coordinate system $\beta_{\tau}: \mathcal{T} \rightarrow \mathbb{R}^{E(\tau)}$. The real numbers $x_{e}=\beta_{\tau}(e) \in \mathbb{R}$ specify the amount of translation along the geodesic associated to the edge $e$ which is required when gluing together the two ideal triangles sharing that geodesic to obtain a given 
quadrilateral in the hyperbolic plane. These are called the shearing coordinates (introduced by Thurston and then considered by Bonahon, Fock et Penner) on the universal Teichmüller space and they provide a homeomorphism $\beta_{\tau}: \mathcal{T} \rightarrow \mathbb{R}^{E(\tau)}$. There is an explicit geometric formula (see also [16, 21]) for the shearing coordinates, as follows. Assume that the union of the two ideal triangles in $\mathbb{H}^{2}$ is the ideal quadrilateral of vertices $p p_{0} p_{-1} p_{\infty}$ and the common geodesic is $p_{\infty} p_{0}$. Then the respective shearing coordinate is the cross-ratio

$$
x_{e}=\left[p, p_{0}, p_{-1}, p_{\infty}\right]=\log \frac{\left(p_{0}-p\right)\left(p_{-1}-p_{\infty}\right)}{\left(p_{\infty}-p\right)\left(p_{-1}-p_{0}\right)}
$$

Let $\tau^{\prime}$ be obtained from $\tau$ by a flip $F_{e}$ and set $\left\{x_{f}^{\prime}\right\}$ for the coordinates associated to $\tau^{\prime}$. The map $\beta_{\tau, \tau^{\prime}}$ : $R^{E\left(\tau^{\prime}\right)} \rightarrow \mathbb{R}^{E(\tau)}$ given by

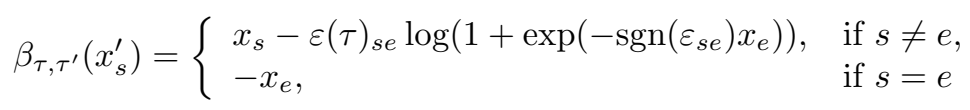

relates the two coordinate systems, namely $\beta_{\tau, \tau^{\prime}} \circ \beta_{\tau^{\prime}}=\beta_{\tau}$.

These coordinate systems provide a contravariant functor $\beta: P t \rightarrow$ Comm from the Ptolemy groupoid $P t$ to the category Comm of commutative topological $*$-algebras over $\mathbb{C}$. We associate to a tessellation $\tau$ the algebra $B(\tau)=C^{\infty}\left(\mathbb{R}^{E(\tau)}, \mathbb{C}\right)$ of smooth complex valued functions on $\mathbb{R}^{E(\tau)}$, with the $*$-structure given by $* f=\bar{f}$. Further to any flip $\left(\tau, \tau^{\prime}\right) \in P t$ one associates the map $\beta_{\tau, \tau^{\prime}}: B\left(\tau^{\prime}\right) \rightarrow B(\tau)$.

The matrices $\varepsilon(\tau)$ have a deep geometric meaning. In fact the bi-vector field

$$
P_{\tau}=\sum_{e, f} \varepsilon(\tau)_{e f} \frac{\partial}{\partial x_{e}} \wedge \frac{\partial}{\partial x_{f}}
$$

written here in the coordinates $\left\{x_{e}\right\}$ associated to $\tau$, defines a Poisson structure on $\mathcal{T}$ which is invariant by the action of the Ptolemy groupoid. The associated Poisson bracket is then given by the formula

$$
\left\{x_{e}, x_{f}\right\}=\varepsilon(\tau)_{e f}
$$

Kontsevich proved that there is a canonical formal quantization of a (finite dimensional) Poisson manifold. The universal Teichmüler space is not only a Poisson manifold but also endowed with a group action and our aim will be an equivariant quantization. Chekhov, Fock and Kashaev (see [11, 28]) constructed an equivariant quantization by means of explicit formulas. There are two ingredients in their approach. First, the Poisson bracket is given by constant coefficients, in any coordinate charts and second, the quantum (di)logarithm.

To any category $C$ whose morphisms are $\mathbb{C}$-vectors spaces one associates its projectivisation $P C$ having the same objects and new morphisms given by $\operatorname{Hom}_{P C}\left(C_{1}, C_{2}\right)=\operatorname{Hom}_{C}\left(C_{1}, C_{2}\right) / U(1)$, for any two objects $C_{1}, C_{2}$ of $C$. Here $U(1) \subset \mathbb{C}$ acts by scalar multiplication. A projective functor into $C$ is actually a functor into $P C$.

Let now $\mathrm{A}^{*}$ be the category of topological *-algebras. Two functors $F_{1}, F_{2}: C \rightarrow \mathrm{A}^{*}$ essentially coincide if there exists a third functor $F$ and natural transformations $F_{1} \rightarrow F, F_{2} \rightarrow F$ providing dense inclusions $F_{1}(O) \hookrightarrow F(O)$ and $F_{2}(O) \hookrightarrow F(O)$, for any object $O$ of $C$.

Definition 3.3. A quantization $\mathcal{T}^{h}$ of the universal Teichmüller space is a family of contravariant projective functors $\beta^{h}: P t \rightarrow \mathrm{A}^{*}$ depending smoothly on the real parameter $h$ such that:

1. The limit $\lim _{h \rightarrow 0} \beta^{h}=\beta^{0}$ exists and essentially coincide with the functor $\beta$.

2. The limit $\lim _{h \rightarrow 0}\left[f_{1}, f_{2}\right] / h$ is defined and coincides with the Poisson bracket on $\mathcal{T}$. Alternatively, for each $\tau$ we have a $\mathbb{C}(h)$-linear (non-commutative) product structure $\star$ on the vector space $C^{\infty}\left(\mathbb{R}^{E(\tau)}, \mathbb{C}(h)\right.$ ) such that

$$
f \star g=f g+h\{f, g\}+o(h)
$$

where $\{f, g\}$ is the Poisson bracket on functions on $\mathcal{T}$ and $\mathbb{C}(h)$ denotes the algebra of smooth $\mathbb{C}$-valued functions on the real parameter $h$. 
We associate to each tessellation $\tau$ the Heisenberg algebra $H_{\tau}^{h}$ which is the topological $*$-algebra over $\mathbb{C}$ generated by the elements $x_{e}, e \in E(\tau)$ subjected to the relations

$$
\left[x_{e}, x_{f}\right]=2 \pi i h \varepsilon(\tau)_{e f}, \quad x_{e}^{*}=x_{e}
$$

We define then $\beta^{h}(\tau)=H_{\tau}^{h}$.

The quantization should associate a homomorphism $\beta^{h}\left(\left(\tau, \tau^{\prime}\right)\right): H_{\tau^{\prime}}^{h} \rightarrow H_{\tau}^{h}$ to each element $\left(\tau, \tau^{\prime}\right) \in P t$. It actually suffices to consider the case when $\left(\tau, \tau^{\prime}\right)$ is the flip $F_{e}$ in the edge $e \in E(\tau)$. Let $\left\{x_{s}^{\prime}\right\}, s \in E\left(\tau^{\prime}\right)$ be the generators of $H_{\tau^{\prime}}^{h}$. We set then

$$
\beta^{h}\left(\left(\tau, \tau^{\prime}\right)\right)\left(x_{s}^{\prime}\right)= \begin{cases}x_{s}-\varepsilon(\tau)_{s e} \phi^{h}\left(-\operatorname{sgn}\left(\varepsilon(\tau)_{s e}\right) x_{e}\right), & \text { if } s \neq e \\ -x_{s}, & \text { if } s=e\end{cases}
$$

Here $\phi^{h}$ is the quantum logarithm function, namely

$$
\phi^{h}(z)=-\frac{\pi h}{2} \int_{\Omega} \frac{\exp (-i t z)}{\operatorname{sh}(\pi t) \operatorname{sh}(\pi h t)} d t
$$

where the contour $\Omega$ goes along the real axes from $-\infty$ to $\infty$ bypassing the origin from above.

Some properties of the quantum logarithm are collected below:

$$
\lim _{h \rightarrow 0} \phi^{h}(z)=\log (1+\exp (z)), \quad \phi^{h}(z)-\phi^{h}(-z)=z, \overline{\phi^{h}(z)}=\phi^{h}(\bar{z}), \quad \frac{\phi^{h}(z)}{h}=\phi^{1 / h}\left(\frac{z}{h}\right)
$$

A convenient way to represent this transformation graphically is to associate to a tessellation its dual binary tree embedded in $\mathbb{H}^{2}$ and to assign to each edge $e$ the respective generator $x_{e}$. Then the action of a flip reads as follows:

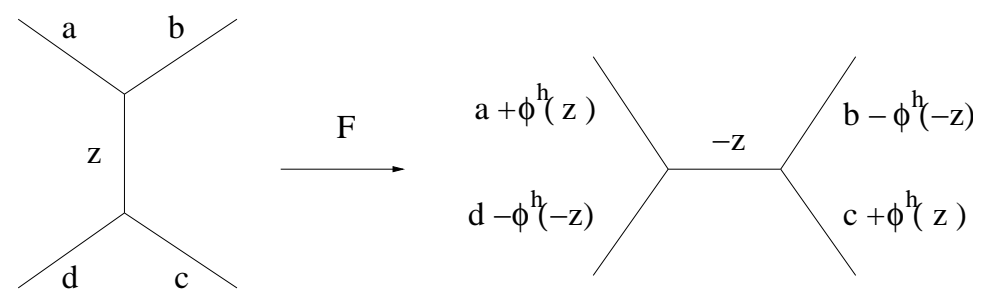

We have then:

Proposition $3.1([11,20])$. The projective functor $\beta^{h}$ is well-defined and it is a quantization of the universal Teichmüller space $\mathcal{T}$.

One proves that $\beta^{h}\left(\left(\tau, \tau^{\prime}\right)\right)$ is independent on the decomposition of the element $\left(\tau, \tau^{\prime}\right)$ as a product of flips. In the classical limit $h \rightarrow 0$ the quantum flip tends to the usual formula of the coordinates change induced by a flip. Thus the first requirement in the definition 3.3 is fulfilled, and the second one is obvious, from the defining relations in the Heisenberg algebra $H_{\tau}^{h}$.

\subsection{The dilogarithmic representation of $T$}

The subject of this section is to give a somewhat self-contained definition of the dilogarithmic representation of the group $T$. The case of general cluster modular groupoids is developed in full detail in [19, 20] and the group $T$ as a cluster modular groupoid is explained in [18.

The quantization of a physical system in quantum mechanics should provide a Hilbert space and the usual procedure is to consider a Hilbert space representation of the algebra from definition 3.3. This is formalized in the notion of representation of a quantum space.

Definition 3.4. A projective *-representation of the quantized universal Teichmüller space $\mathcal{T}^{h}$, specified by the functor $\beta^{h}: P t \rightarrow \mathrm{A}^{*}$, consists of the following data: 
1. A projective functor Pt $\rightarrow$ Hilb to the category of Hilbert spaces. In particular, one associates a Hilbert space $\mathcal{L}_{\tau}$ to each tessellation $\tau$ and a unitary operator $K_{\left(\tau, \tau^{\prime}\right)}: \mathcal{L}_{\tau} \rightarrow \mathcal{L}_{\tau^{\prime}}$, defined up to a scalar of absolute value 1.

2. A*-representation $\rho_{\tau}$ of the Heisenberg algebra $H_{\tau}^{h}$ in the Hilbert space $\mathcal{L}_{\tau}$, such that the operators $\mathbf{K}_{\left(\tau, \tau^{\prime}\right)}$ intertwine the representations $\rho_{\tau}$ and $\rho_{\tau^{\prime}}$ i.e.

$$
\rho_{\tau}(w)=\mathbf{K}_{\left(\tau, \tau^{\prime}\right)}^{-1} \rho_{\tau^{\prime}}\left(\beta^{h}\left(\left(\tau, \tau^{\prime}\right)\right)(w)\right) \mathbf{K}_{\left(\tau, \tau^{\prime}\right)}, w \in H_{\tau}^{h}
$$

The classical Heisenberg $*$-algebra $H$ is generated by $2 n$ elements $x_{s}, y_{s}, 1 \leq s \leq n$ and relations

$$
\left[x_{s}, y_{s}\right]=2 \pi i h, \quad\left[x_{s}, y_{t}\right]=0, \text { if } s \neq t, \quad\left[x_{s}, x_{t}\right]=\left[y_{s}, y_{t}\right]=0 \text {, for all } s, t
$$

with the obvious $*$-structure. The single irreducible integrable $*$-representation $\rho$ of $H$ makes it act on the Hilbert space $L^{2}\left(\mathbb{R}^{n}\right)$ by means of the operators:

$$
\rho\left(x_{s}\right) f\left(z_{1}, \ldots, z_{n}\right)=z_{s} f\left(z_{1}, \ldots, z_{n}\right), \quad \rho\left(y_{s}\right)=-2 \pi i h \frac{\partial f}{\partial z_{s}}
$$

The Heisenberg algebras $H_{\tau}^{h}$ are defined by commutation relations with constant coefficients and hence their representations can be constructed by selecting a Lagrangian subspace in the generators $x_{s}-$ called a polarization - and let the generators act as linear combinations in the operators $\rho\left(x_{s}\right)$ and $\rho\left(y_{s}\right)$ above.

The Stone von Neumann theorem holds true then for these algebras. Specifically, there exists an unique unitary irreducible Hilbert space representation of given central character that is integrable i.e. which can be integrated to the corresponding Lie group. Notice that there exist in general also non-integrable unitary representations.

In particular we obtain representations of $H_{\tau}^{h}$ and $H_{\tau^{\prime}}^{h}$. The uniqueness of the representation yields the existence of an intertwinner $\mathbf{K}_{\left(\tau, \tau^{\prime}\right)}$ (defined up to a scalar) between the two representations. However, nor the Hilbert spaces neither the representations $\rho_{\tau}$ are not canonical, as they depend on the choice of the polarization.

We will give below the construction of a canonical representation when the quantized Teichmüller space is replaced by its double. We need first to switch to another system of coordinates, coming from the cluster $\mathcal{A}$-varieties. Define, after Penner (see [31]) the universal decorated Teichmüller space $\mathcal{A}$ to be the space of all marked tessellations endowed with one horocycle for each vertex (decoration). Alternatively (see [17]), $\mathcal{A}$ is the set of positive real points of the cluster $\mathcal{A}$-space related to the previous set of seeds.

Each tessellation $\tau$ yields a coordinate system $\alpha_{\tau}: \mathcal{A} \rightarrow \mathbb{R}^{E(\tau)}$ which associates to the edge $e$ of $\tau$ the coordinate $a_{e}=\alpha_{\tau}(e) \in \mathbb{R}$. The number $\alpha_{\tau}(e)$ is the algebraic distance between the two horocycles on $\mathbb{H}^{2}$ centered at vertices of $e$, measured along the geodesic associated to $e$. These are the so-called lambda coordinates of Penner.

There is a canonical map $p: \mathcal{A} \rightarrow \mathcal{T}$ (see [31, Proposition 3.7 and [17]) such that, in the coordinate systems induced by a tessellation $\tau$, the corresponding map $p_{\tau}: \mathbb{R}^{(E(\tau)} \rightarrow \mathbb{R}^{E(\tau)}$ is given by

$$
p_{\tau}\left(\sum_{t \in E(\tau)} \varepsilon(\tau)_{s t} a_{t}\right)=x_{s}
$$

Let $\left(\tau, \tau^{\prime}\right)$ be the flip on the edge $e$ and set $a_{s}^{\prime}$ be the coordinates system associated to $\tau^{\prime}$. Then the flip induces the following change of coordinates:

$$
\alpha_{\tau, \tau^{\prime}}\left(a_{s}\right)= \begin{cases}a_{s}, & \text { if } s \neq e \\ -a_{e}+\log \left(\exp \left(\sum_{t ; \varepsilon(\tau)_{e t}>0} \varepsilon(\tau)_{e t} a_{t}\right)+\exp \left(-\sum_{t ; \varepsilon(\tau)_{e t}<0} \varepsilon(\tau)_{e t} a_{t}\right)\right), & \text { if } s=e\end{cases}
$$

It can be verified that $p_{\tau}$ are compatible with the action of the Ptolemy groupoid on the respective coordinates.

The vector space $\mathcal{L}_{\tau}$ is defined as the space of square integrable functions with finite dimensional support on $\mathcal{A}$ with respect to the $\alpha_{\tau}$ coordinates i.e. the functions $f: \mathbb{R}^{E(\tau)} \rightarrow \mathbb{C}$, with support contained into some 
$R^{F} \times\{0\} \subset R^{E(\tau)}$, for some finite subset $F \subset E(\tau)$. The coordinates on $\mathbb{R}^{E(\tau)}$ are the $a_{e}, e \in E(\tau)$. The function $f$ is square integrable if

$$
\int_{\mathbb{R}^{F}}|f|^{2} \bigwedge_{e \in F} d a_{e}<\infty
$$

for any such $F$ as above. Let $f, g \in \mathcal{L}_{\tau}$. Then let $\mathbb{R}^{F} \times\{0\}$ contain the intersection of their supports. Choose $F$ minimal with this property. Then the scalar product

$$
\langle f, g\rangle=\int_{\mathbb{R}^{F}} f(a) \overline{g(a)} \bigwedge_{e \in F} d a_{e}
$$

makes $\mathcal{L}_{\tau}$ a Hilbert space.

To define the intertwining operator $\mathbf{K}$ we set now:

$$
G_{e}\left(\left(a_{s}\right)_{s \in F}\right)=\int \exp \left(\int_{\Omega} \frac{\exp \left(i t \sum_{s \in F} \varepsilon(\tau)_{e s} a_{s}\right) \sin (t c)}{2 i \operatorname{sh}(\pi t) \operatorname{sh}(\pi h t)} \frac{d t}{t}+\frac{c}{\pi i h}\left(\sum_{s ; \varepsilon(\tau)_{e s}<0} \varepsilon(\tau)_{e s} a_{s}+a_{e}\right)\right) d c
$$

The key ingredient in the construction of this function is the quantum dilogarithm (going back to Barnes ([1]) and used by Baxter (4]) and Faddeev ([14])):

$$
\Phi^{h}(z)=\exp \left(-\frac{1}{4} \int_{\Omega} \frac{\exp (-i t z)}{\operatorname{sh}(\pi t) \operatorname{sh}(\pi h t)} \frac{d t}{t}\right)
$$

where the contour $\Omega$ goes along the real axes from $-\infty$ to $\infty$ bypassing the origin from above.

Some properties of the quantum dilogarithm are collected below:

$$
\begin{gathered}
2 \pi i h d \log \Phi^{h}(z)=\phi^{h}(z), \lim _{\Re z \rightarrow-\infty} \Phi^{h}(z)=1 \\
\lim _{h \rightarrow 0} \Phi^{h}(z) / \exp \left(-\operatorname{Li}_{2}(-\exp (z))\right)=2 \pi i h, \text { where } \operatorname{Li}_{2}(z)=\int_{0}^{z} \log (1-t) d t \\
\Phi^{h}(z) \Phi^{h}(-z)=\exp \left(\frac{z^{2}}{4 \pi i h}\right) \exp \left(-\frac{\pi i}{12}\left(h+h^{-1}\right)\right), \overline{\left.\Phi^{h}(z)\right)}=\left(\Phi^{h}(\bar{z})\right)^{-1}, \Phi^{h}(z)=\Phi^{1 / h}\left(\frac{z}{h}\right)
\end{gathered}
$$

Let now $f \in \mathcal{L}_{\tau}$, namely some $f: \mathbb{R}^{F} \times\{0\} \rightarrow \mathbb{C}$. Consider $\left(\tau, \tau^{\prime}\right)$ be the flip $F_{e}$ on the edge $e$. Let $a_{s}, s \in F$ be the coordinates in $\mathbb{R}^{F}$. If $e \notin F$ then we set

$$
\mathbf{K}_{\left(\tau, \tau^{\prime}\right)}=1
$$

If $e \in F$ then the coordinates associated to $\tau^{\prime}$ are $a_{s}, s \neq e$ and $a_{e}^{\prime}$. Set then

$$
\left(\mathbf{K}_{\left(\tau, \tau^{\prime}\right)} f\right)\left(\left(a_{s}, s \in F, f \neq e, a_{e}^{\prime}\right)=\int G_{e}\left(\left(a_{s}\right)_{s \in F, s \neq e}, a_{e}+a_{e}^{\prime}\right) f\left(\left(a_{s}\right)_{s \in F}\right) d a_{s}\right.
$$

The last piece of data is the representation of the Heisenberg algebra $H_{\tau}^{h}$ in the Hilbert space $\mathcal{L}_{\tau}$. We can actually do better, namely to enhance the space with a bimodule structure. Set

$$
\begin{gathered}
\rho_{\tau}^{-}\left(x_{s}\right)=-\pi i h \frac{\partial}{\partial a_{s}}+\sum_{t} \varepsilon(\tau)_{s t} a_{t} \\
\rho_{\tau}^{+}\left(x_{s}\right)=\pi i h \frac{\partial}{\partial a_{s}}+\sum_{t} \varepsilon(\tau)_{s t} a_{t}
\end{gathered}
$$

Then $\rho_{\tau}^{-}$gives a left module and $\rho_{\tau}^{+}$a right module structure on $\mathcal{L}_{\tau}$ and the two actions commute. We have then:

Proposition $3.2([11,20,19])$. The data $\left(\mathcal{L}_{\tau}, \rho_{\tau}^{ \pm}, \mathbf{K}_{\left(\tau, \tau^{\prime}\right)}\right)$ is a projective $*$-representation of the quantized universal Teichmüller space. 
We call it the dilogarithmic representation of the Ptolemy groupoid. The proof of this result is given in [19] and a particular case is explained with lots of details in [26.

The last step in our construction is to observe that a representation of the Ptolemy groupoid Pt induces a representation of the Ptolemy-Thompson group $T$ by means of an identification of the Hilbert spaces $\mathcal{L}_{\tau}$ for all $\tau$.

Projective representations are equivalent to representations of central extensions by means of the following well-known procedure. To a general group $G$, Hilbert space $V$ and homomorphism $A: G \rightarrow P G L(V)$ we can associate a central extension $\widetilde{G}$ of $G$ by $\mathbb{C}^{*}$ which resolves the projective representation $A$ to a linear representation $\widetilde{A}: \widetilde{G} \rightarrow G L(V)$. The extension $\widetilde{G}$ is the pull-back on $G$ of the canonical central $\mathbb{C}^{*}$-extension $G L(V) \rightarrow P G L(V)$.

However the central extension which we consider here is a subgroup of the $\mathbb{C}^{*}$-extension defined above, obtained by using a particular section over $G$. Let us write $G=F / R$ as the quotient of the free group $F$ by the normal subgroup $R$ generated by the relations. Then our data consists in a homomorphism $\bar{A}: F \rightarrow G L(V)$ with the property that $\bar{A}(r) \in \mathbb{C}^{*}$, for each relation $r \in R$, so that $\bar{A}$ induces $A: G \rightarrow P G L(V)$. This data will be called an almost-linear representation, in order to distinguish it from a projective representation of G.

The central extension $\widehat{G}$ of $G$ associated to $\bar{A}$ is $\widehat{G}=F /(\operatorname{ker} \bar{A} \cap R)$, namely the smallest central extension of $S$ resolving the projective representation $A$ to a linear representation compatible with $\bar{A}$. Then $\widehat{G}$ is a central extension of $G$ by the subgroup $\bar{A}(R) \subset \mathbb{C}^{*}$ and hence it is naturally a subgroup of $\widetilde{G}$. In other terms $\bar{A}$ determines a projective representation $A$ and a section over $G$ whose associated 2-cocycle takes values in $\bar{A}(R)$ and which describes the central extension $\widehat{G}$.

Now, the intertwinner functor $\mathbf{K}$ is actually an almost-linear representation (in the obvious sense) of the Ptolemy groupoid and thus induces an almost-linear representation of the Ptolemy-Thompson group $T$ into the unitary group. We can extract from [19] the following results (see also the equivalent construction at the level of Heisenberg algebras in [3]):

Proposition 3.3. The dilogarithmic almost-linear representation $\mathbf{K}$ has the following properties:

1. Images of disjoint flips in $\widehat{T}$ commute with each other;

2. The square of a flip is the identity;

3. The composition of the lifts of the five flips from the pentagon relation below is $\exp (2 \pi i h)$ times the symmetry permuting the two edges coordinates.

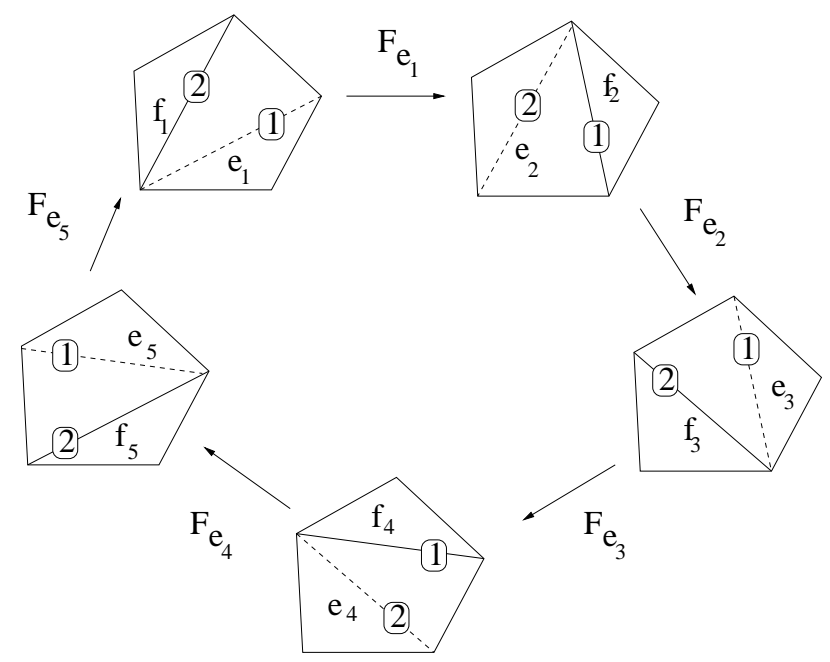

Proof. The first condition is that images by $K$ of flips on disjoint edges should commute. This is obvious by the explicit formula for $K$. The second and third conditions are proved in [19]. 
Therefore the image by $\mathbf{K}$ of relations of the Ptolemy groupoid into $\mathbb{C}^{*}$ is the subgroup $U$ generated by $\exp (2 \pi i h)$. We can view the pentagon relation in the Ptolemy-Thompson group $T$ as a pentagon relation in the Ptolemy groupoid Pt. Thus the image by $\mathbf{K}$ of relations of the Ptolemy-Thompson group $T$ into $\mathbb{C}^{*}$ is also the subgroup $U$. In particular the associated 2-cocycle takes values in $U$. If $h$ is a formal parameter or an irrational real number we obtain then a 2-cocycle with values in $\mathbb{Z}$.

Definition 3.5. The dilogarithmic central extension $\widehat{T}$ is the central extension of $T$ by $\mathbb{Z}$ associated to the dilogarithmic almost-linear representation $\mathbf{K}$ of $T$, or equivalently, to the previous 2-cocycle.

\subsection{Identifying the two central extensions of $T$}

The main result of this section is the following:

Proposition 3.4. The dilogarithmic extension $\widehat{T}$ is identified to $T_{\mathrm{ab}}^{*}$.

Proof. The main step is to translate the properties of the dilogarithmic representation of the Ptolemy groupoid in terms of the Ptolemy-Thompson group. Since $\widehat{T}$ is a central extension of $T$ it is generated by the lifts $\widehat{\alpha}, \widehat{\beta}$ of $\alpha$ and $\beta$ together with the generator $z$ of the center. Let us see what are the relations arising in the group $\widehat{T}$. According to proposition 3.3 lifts of disjoint flips should commute. By a simple computation we can show that the elements $\beta \alpha \beta, \alpha^{2} \beta \alpha \beta \alpha^{2}$ and $\alpha^{2} \beta \alpha^{2} \beta \alpha \beta \alpha^{2} \beta^{2} \alpha^{2}$ act as disjoint flips on the Farey triangulation. In particular we have the relations

$$
\left[\widehat{\beta} \widehat{\alpha} \widehat{\beta}, \widehat{\alpha}^{2} \widehat{\beta} \widehat{\alpha} \widehat{\beta} \widehat{\alpha}^{2}\right]=\left[\widehat{\beta} \widehat{\alpha} \widehat{\beta}, \widehat{\alpha}^{2} \widehat{\beta} \widehat{\alpha}^{2} \widehat{\beta} \widehat{\alpha} \widehat{\beta} \widehat{\alpha}^{2} \widehat{\beta}^{2} \widehat{\alpha}^{2}\right]=1
$$

satisfied in $\widehat{T}$. Moreover, by construction we have also

$$
\widehat{\beta}^{3}=\widehat{\alpha}^{4}=1
$$

meaning that the $\widehat{\alpha}$ is still periodic of order 4 while $\widehat{\beta}$ is not deformed.

Eventually the only non-trivial lift of relations comes from the pentagon relation $(\widehat{\beta} \widehat{\alpha})^{5}$. The element $(\widehat{\beta} \widehat{\alpha})^{5}$ is actually the permutation of the two edges in the pentagon times the composition of the five flips. The pentagon equation is not anymore satisfied but proposition 3.3 shows that the dilogarithmic image of $(\widehat{\beta} \widehat{\alpha})^{5}$ is a scalar operator. Since $z$ is the generator of the kernel $\mathbb{Z}$ of $\widehat{T} \rightarrow T$ it follows that the the lift of the pentagon equation from $T$ to $\widehat{T}$ is given by

$$
(\widehat{\beta} \widehat{\alpha})^{5}=z
$$

According to proposition 2.1 all relations presenting $T_{\mathrm{ab}}^{*}$ are satisfied in $\widehat{T}$. Since $\widehat{T}$ is a nontrivial central extension of $T$ by $\mathbb{Z}$ it follows that the groups are isomorphic.

Remark 3.1. The key-point in the proof above is that all pentagon relations in $P t$ are transformed in a single pentagon relation in $T$ and thus the scalars associated to the pentagons in $P t$ should be the same.

\section{Classification of central extensions of the group $T$}

\subsection{The family $T_{n, p, q, r}$ of central extensions}

Our main concern here is to identify the cohomology classes of all central extensions of $T$ in $H^{2}(T)$. Before doing that we consider a series of central extensions $T_{n, p, q, r, s}$ of $T$ by $\mathbb{Z}$, having properties similar to those of $\widehat{T}$.

Definition 4.1. The group $T_{n, p, q, r, s}$, is presented by the generators $\bar{\alpha}, \bar{\beta}, z$ and the relations:

$$
\begin{gathered}
(\bar{\beta} \bar{\alpha})^{5}=z^{n} \\
\bar{\alpha}^{4}=z^{p} \\
\bar{\beta}^{3}=z^{q} \\
{\left[\bar{\beta} \bar{\alpha} \bar{\beta}, \bar{\alpha}^{2} \bar{\beta} \bar{\alpha} \bar{\beta} \bar{\alpha}^{2}\right]=z^{r}} \\
{\left[\bar{\beta} \bar{\alpha} \bar{\beta}, \bar{\alpha}^{2} \bar{\beta} \bar{\alpha}^{2} \bar{\beta} \bar{\alpha} \bar{\beta} \bar{\alpha}^{2} \bar{\beta}^{2} \bar{\alpha}^{2}\right]=z^{s}} \\
{[\bar{\alpha}, z]=[\bar{\beta}, z]=1}
\end{gathered}
$$

Let us denote $T_{n, p, q, r}=T_{n, p, q, r, 0}$ and $T_{n, p, q}=T_{n, p, q, 0,0}$. 
According to 23 we can identify $\widehat{T}$ with $T_{1,0,0}$. In fact the group $T^{*}$ is split over the smaller Thompson group $F \subset T$ and thus $\widehat{T}$ is split over $F$. Further $F$ is generated by the elements $\beta^{2} \alpha$ and $\beta \alpha^{2}$ and thus relations of $F$ are precisely given by the commutation relations above. Thus the last two relations hold true, while $z$ is central and thus $\widehat{T}$ is given by the presentation above.

Remark 4.1. We considered in 23] the twin group $T^{\sharp}$ and gave a presentation of it. Then, using a similar procedure there is a group obtained from $T^{\sharp}$ by abelianizing the kernel $B_{\infty}$, which is identified actually to $T_{3,1,0}$.

Proposition 4.1. Central extensions of $T$ by $\mathbb{Z}$ are exhausted by the set of extensions $T_{n, p, q, r}$.

Proof. Consider the relations in $T_{n, p, q, r, s}$ other than

$$
\left[\bar{\beta} \bar{\alpha} \bar{\beta}, \bar{\alpha}^{2} \bar{\beta} \bar{\alpha}^{2} \bar{\beta} \bar{\alpha} \bar{\beta} \bar{\alpha}^{2} \bar{\beta}^{2} \bar{\alpha}^{2}\right]=z^{s}
$$

It suffices to see that these relations already force

$$
\left[\bar{\beta} \bar{\alpha} \bar{\beta}, \bar{\alpha}^{2} \bar{\beta} \bar{\alpha}^{2} \bar{\beta} \bar{\alpha} \bar{\beta} \bar{\alpha}^{2} \bar{\beta}^{2} \bar{\alpha}^{2}\right]=1
$$

The commutator $\left[\beta \alpha \beta, \alpha^{2} \beta \alpha^{2} \beta \alpha \beta \alpha^{2} \beta^{2} \alpha^{2}\right]$ is the trivial element of $T$ and thus it leads by means of Hopf theorem to a 2-cycle on $T$, given by the formula:

$$
\delta=\left(\beta \alpha \beta, \alpha^{2} \beta \alpha^{2} \beta \alpha \beta \alpha^{2} \beta^{2} \alpha^{2}\right)-\left(\alpha^{2} \beta \alpha^{2} \beta \alpha \beta \alpha^{2} \beta^{2} \alpha^{2}, \beta \alpha \beta\right)
$$

However the commutator above can be written as a commutator in the subgroup $F$ as we already remarked that

$$
\left[\beta \alpha \beta, \alpha^{2} \beta \alpha^{2} \beta \alpha \beta \alpha^{2} \beta^{2} \alpha^{2}\right]=\left[A B^{-1}, A^{-2} B A^{2}\right]
$$

where $A=\beta \alpha^{2}$ and $B=\beta^{2} \alpha$ are the generators of $F$. Moreover the last commutator defines the 2-cycle

$$
\epsilon=\left(A B^{-1}, A^{-2} B A^{2}\right)-\left(A^{-2} B A^{2}, A B^{-1}\right)
$$

in $H_{2}(F)$ and the inclusion $i: F \subset T$ sends the class of $[\epsilon]$ into $i_{*}([\epsilon])=[\delta]$. However, it is known from 25] that $[\epsilon]$ is in the kernel of $i_{*}: H_{2}(F) \rightarrow H_{2}(T)$ and thus $[\delta]=0$. This shows that $\left\langle c_{T_{n, p, q, r, s}},[\epsilon]\right\rangle=0$, where $\langle\rangle:, H^{2}(T) \times H_{2}(T) \rightarrow \mathbb{Z}$ is the obvious pairing. Thus $T_{n, p, q, r, s}$ splits over the subgroup generated by $\beta \alpha \beta$ and $\alpha^{2} \beta \alpha^{2} \beta \alpha \beta \alpha^{2} \beta^{2} \alpha^{2}$. Thus up to changing each one of the lifts $\bar{\alpha}, \bar{\beta} \in T_{n, p, q, r, s}$ of $\alpha, \beta$ by a central factor we have

$$
\left[\bar{\beta} \bar{\alpha} \bar{\beta}, \bar{\alpha}^{2} \bar{\beta} \bar{\alpha}^{2} \bar{\beta} \bar{\alpha} \bar{\beta} \bar{\alpha}^{2} \bar{\beta}^{2} \bar{\alpha}^{2}\right]=1
$$

However, if we chose another lifts then central factors will cancel each other in the commutator above and thus the identity above holds true for any choice of the lifts. In particular we have $s=0$.

The aim of this chapter is to prove theorem 1.2, namely to compute the class $c_{T_{n, p, q, r}} \in H^{2}(T)$ of the extension $T_{n, p, q, r}$. We denote by $\chi(n, p, q, r)$ the coefficient of $\chi$ and $\alpha(n, p, q, r)$ the coefficient of $\alpha$ in $c_{T_{n, p, q, r}}$.

\subsection{Computing $\alpha(n, p, q, r)$}

Proposition 4.2. We have $\alpha(n, p, q, r)=r$.

Proof. Since the commutator $\left[\beta \alpha \beta, \alpha^{2} \beta \alpha \beta \alpha^{2}\right]$ is the identity in $T$ it gives rise to a 2-cycle in homology given by

$$
\mu=\left(\beta \alpha \beta, \alpha^{2} \beta \alpha \beta \alpha^{2}\right)-\left(\alpha^{2} \beta \alpha \beta \alpha^{2}, \beta \alpha \beta\right)
$$

and hence representing a class $[\mu] \in H_{2}(T)$. As in the proof of Proposition $4.1[\mu]=i_{*}([\eta])$ where

$$
\eta=\left(A B^{-1}, A^{-1} B A\right)-\left(A^{-1} B A, A B^{-1}\right)
$$

is the 2-cycle on $F$ associated to the commutator $\left[A B^{-1}, A^{-1} B A\right]$.

We claim that

Lemma 4.1. $\langle\alpha,[\mu]\rangle=1$, where $\langle\rangle:, H^{2}(T) \times H_{2}(T) \rightarrow \mathbb{Z}$ is the obvious pairing. 
Proof. Consider the discrete Godbillon-Vey 2-cocycle $\overline{g v}: T \times T \rightarrow \mathbb{Z}$ defined by the formula

$$
\overline{g v}(g, h)=\sum_{x \in S^{1}} \operatorname{det}\left(\begin{array}{cc}
\log _{2} h_{r}^{\prime}(x) & \log _{2}(g \circ h)_{r}^{\prime}(x) \\
h^{\prime \prime}(x) & (g \circ h)^{\prime \prime}(x)
\end{array}\right)
$$

Here $\gamma^{\prime \prime}$ stands for

$$
\gamma^{\prime \prime}(x)=\log _{2} \gamma_{r}^{\prime}(x)-\log _{2} \gamma_{l}^{\prime}(x)
$$

where $\gamma_{r}^{\prime}, \gamma_{l}^{\prime}$ are the right respectively left derivatives of $\gamma$. Notice that the derivative of $\gamma \in T$ is a locally constant function having only finitely many discontinuity points.

It is well-known (see [25]) that the 2 -cocycle $\overline{g v}$ represents $2 \alpha$ in cohomology. A direct computation using this cocycle shows that $\overline{g v}(\mu)=2$ and hence proving the claim.

We have

$$
\left\langle c_{T_{n, p, q, r}}, i_{*}([\eta])\right\rangle=\left\langle c_{T_{n, p, q, r}} \mid{ }_{F},[\eta]\right\rangle=\left\langle c_{F_{r}},[\eta]\right\rangle
$$

where $F_{r}$ is the central extension of $F$ given by

$$
F_{r}=\left\langle A, B, z ;\left[A B^{-1}, A^{-1} B A\right]=z^{r},\left[A B^{-1}, A^{-2} B A^{2}\right]=1,[z, A]=[z, B]=1\right\rangle
$$

According to the arguments from the proof of Proposition 4.1 these extensions exhaust the set of all central extensions of $F$ that arise from central extensions of $T$.

On the other hand we have

$$
\begin{aligned}
\left.\left\langle c_{T_{n, p, q, r}},[\mu]\right)\right\rangle & =\left\langle\alpha(n, p, q, r) \alpha+\chi(n, p, q, r) \chi, i_{*}[\eta]\right\rangle=\left\langle\left.\alpha(n, p, q, r) \alpha\right|_{F}+\chi(n, p, q, r) \chi \mid F,[\eta]\right\rangle \\
& =\alpha(n, p, q, r)\langle\alpha \mid F,[\eta]\rangle=\alpha(n, p, q, r)
\end{aligned}
$$

because the image of $\chi$ in $H^{2}(F)$ vanishes.

Observe that $F_{r}$ does not depend on $n, p, q$ and thus its class does not depend either. This shows that $\alpha(n, p, q, r)$ is a function on $r$. This function is computed through the choice of a setwise section in the projection $F_{r} \rightarrow F$, which is further evaluated to a fixed 2-cycle $\eta$ and thus it should be a linear function with integer coefficients and without constant terms. This implies that $\alpha(n, p, q, r)=\lambda r$, for $\lambda \in \mathbb{Z}$.

Eventually by choosing explicit lifts of the elements $A B^{-1}$ and $A^{-1} B A$ (and their products in whatever order) in $F_{r}$ we can evaluate $\left\langle c_{F_{r}},[\eta]\right\rangle=r$.

Corollary 4.1. The extensions $T_{n, p, q, r}$ describe all central extensions of $T$ by $\mathbb{Z}$.

The extensions $T_{n, p, q, 0}$ describe all central extensions of $T$ by $\mathbb{Z}$ associated to multiples of the Euler class, and all of them split over $F$.

Proof. The groups $T_{n, p, q, 0}$ are extensions of $T$ by a cyclic group. Moreover any central extension of $T$ has the form $T_{n, p, q, r}$. The formula for $\alpha(n, p, q, r)$ shows that $r=0$ if we want that its class be a multiple of the Euler class. Further the set of central extensions of $T$ by $\mathbb{Z}$ up to isomorphism over $T$ are in one-toone correspondence with the classes of $H^{2}(T)$. In particular any multiple of $\chi$ should be realized by one extension by $\mathbb{Z}$. Now, for given $m \in \mathbb{Z}$ there exists only one isomorphism type of $T_{n, p, q, 0}$ for which $n, p, q$ are solutions of $12 n-15 p-20 q=m$. This follows from the uniqueness of solutions up to equivalence $p \sim p+4 x$, $q \sim q+3 y, n \sim n+5 x+5 y$. Thus the respective extension must have kernel $\mathbb{Z}$.

\subsection{The Greenberg-Sergiescu extension $\mathcal{A}_{T}$}

We know the value of $\chi(n, p, q, r)$ for $r=0$, but in order to find the coefficient of $r$ we need to know explicitly central extensions with nontrivial Godbillon-Vey class. Fortunately, Greenberg and Sergiescu in [27] had constructed such an extension of $T$ by $B_{\infty}$. The main difficulty in analyzing this extension comes from the fact that there are several perspectives for analyzing the group $T$, either as a group of dyadic piecewise affine homeomorphisms of $S^{1}$ or else as a group $\operatorname{PPSL}(2, \mathbb{Z})$ of piecewise $P S L(2, \mathbb{Z})$ automorphisms of the circle at infinity. If we plug in the discrete Godbillon-Vey then the formulas from [25, 27] use the first point of view. If we are seeking for the mapping class group perspective it is the second point of view which is manifest 
and there is no direct relationship between this and the former one. The key-point in the calculations below is to pass from one perspective to the other. We have therefore to give a detailed account of the group $\mathcal{A}_{T}$, following Kapoudjian and Sergiescu [29].

We will use the mapping class group description of the Ptolemy-Thompson group but we will enlarge the surface. We follow closely [29, 23].

The surface $D$ occurs in the process of understanding the almost action of $T$ on the infinite binary tree. Recall that an almost automorphism of a tree is a map sending the complement of a finite tree isomorphically on the complement of a finite tree. The action of $T$ acting as mapping class group of $D$ induces an almost action on the binary tree. This point of view emphasize the realization of $T$ as the group $P P S L(2, \mathbb{Z})$.

We can think of $T$ as the group of dyadic piecewise affine homeomorphisms of $S^{1}$. This will lead to another, more subtle, way to construct an action of $T$ on a regular tree. Let $\mathcal{T}$ be the rooted binary tree with one finite leaf, obtained by splitting one edge of the usual binary tree at some vertex (the root) and attaching one more edge. Label the vertices and edges (see [10]) of $\mathcal{T}$ inductively as follows. The leaf is labeled $0 \sim 1$ and the edge joining it with the root by $[0,1]$. The root is labeled $\frac{1}{2}$, its left descending edge is labeled $\left[0, \frac{1}{2}\right]$ and the right descending one $\left[\frac{1}{2}, 1\right]$. Further if an edge is labeled $\left[\frac{k}{2^{n}}, \frac{k+1}{2^{n}}\right]$, then its bottom vertex is labeled $\frac{2 k+1}{2^{n+1}}$ and the two edges issued from it are labeled, the left one $\left[\frac{k}{2^{n}}, \frac{2 k+1}{2^{n+1}}\right]$ and the right one $\left[\frac{2 k+1}{2^{n+1}}, \frac{k+1}{2^{n}}\right]$ respectively.

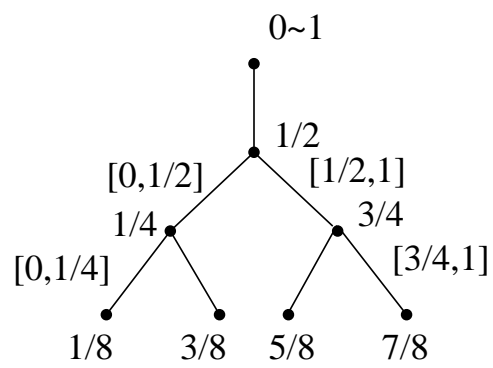

The almost action of $T$ on $\mathcal{T}$ could be read off from the description of elements of $T$ as pairs of stable binary trees. Alternatively, we identify elements of $T$ as dyadic piecewise affine homeomorphisms of $S^{1}=[0,1] / 0 \sim$ 1. The action of such a homeomorphism induces a bijection of the set of vertices of $\mathcal{T}$ (identified to their labels). This bijection is an almost automorphism of the tree $\mathcal{T}$.

Consider next the tree $\mathcal{E} T$ obtained from $\mathcal{T}$ by adjoining a pending line (with infinitely many vertices on it) to each vertex of $\mathcal{T}$.

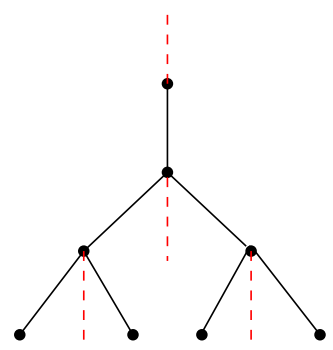

There is an obvious extension of the almost action of $T$ from $\mathcal{T}$ to $\mathcal{E} T$. However there exists a more interesting one as it was discovered in [27].

Definition 4.2. Let $\frac{\mathbb{Z}\left[\frac{1}{2}\right]}{\mathbb{Z}} \subset S^{1}$ denote the set of images of dyadic numbers. Assume that $T$ is identified with the subgroup of $\mathrm{Homeo}^{+}\left(S^{1}\right)$ of piecewise linear homeomorphisms. A cocycle is a map $K: T \times \frac{\mathbb{Z}\left[\frac{1}{2}\right]}{\mathbb{Z}} \rightarrow \mathbb{Z}$ satisfying the following conditions:

1. For any $\gamma \in T, K(\gamma, x)$ vanishes for all but finitely many points $x \in \frac{\mathbb{Z}\left[\frac{1}{2}\right]}{\mathbb{Z}}$ and

$$
\sum_{x \in \frac{\mathbb{Z}\left[\frac{1}{2}\right]}{\mathbb{Z}}} K(\gamma, x)=0
$$


2. $K(\gamma \delta, w)=K(\gamma, \delta(w))+K(\delta, w)$, for all $\gamma, \delta \in T, w \in \frac{\mathbb{Z}\left[\frac{1}{2}\right]}{\mathbb{Z}}$.

For any cocycle $K: T \times \frac{\mathbb{Z}\left[\frac{1}{2}\right]}{\mathbb{Z}} \rightarrow \mathbb{Z}$ we can associate such an action. Let $\gamma \in T$. Then $\gamma$ induces a bijection denoted by the same letter between the vertices of the rooted tree $\mathcal{T}$, which were identified with $\frac{\mathbb{Z}\left[\frac{1}{2}\right]}{\mathbb{Z}}$. This bijection induces an almost automorphism of the tree $\mathcal{T}$. Moreover, let $n=\max _{x \in \frac{\mathbb{Z}\left[\frac{1}{2}\right]}{\mathbb{Z}}}|K(\gamma, x)|$. If $v \in \frac{\mathbb{Z}\left[\frac{1}{2}\right]}{\mathbb{Z}}$ is a vertex of $\mathcal{T}$ let $f_{v}$ denote the pending line at $v$ and $f_{v}^{\geq n} \subset f_{v}$ be the subtree of those points at distance at least $n$ from $v$. We define the almost action of $\gamma$ on $\mathcal{E} T$ as being the unique isometric bijection from $f_{v}^{\geq n}$ to $f_{\gamma(v)}^{\geq n}$.

Definition 4.3. The enhanced ribbon tree ED is obtained by thickening $\mathcal{E} T$ in the plane and $E D^{*}$ by puncturing $E D$ along the vertices of the pending lines. Notice that the vertices of $\mathcal{T}$ are not among the punctures.

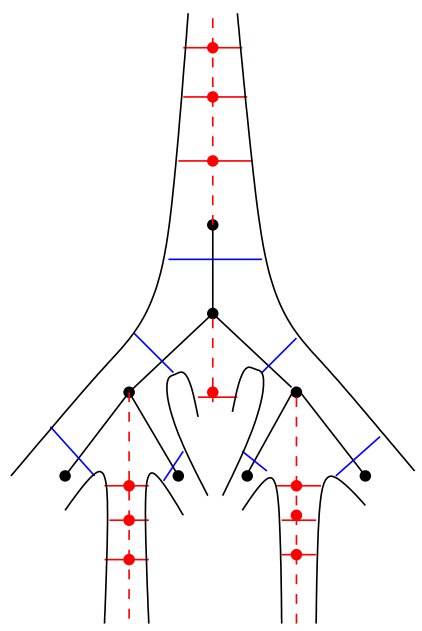

The canonical rigid structures of $E D$ and $E D^{*}$ are those from the picture above which decomposes $E D$ into (punctured) octagons centered at vertices of $\mathcal{T}$ and (punctured) squares along the pending lines at vertices of $\mathcal{T}$.

Admissible sub-surfaces of ED or ED* are connected finite unions of elementary pieces.

Given a cocycle $K$ one finds that the almost action of $T$ on $\mathcal{E} T$ induces an embedding of the group $T$ into the asymptotic mapping class group $\mathcal{M}(E D)$ with the given structure. Specifically, define the content of an admissible subsurface $\Sigma \subset E D$ to be the number of squares it contains. Then $T$ is the group of mapping classes that preserve the content i.e. those mapping classes of homeomorphisms $\varphi$ for which $\varphi(\Sigma)$ and $\Sigma$ have the same content for any admissible $\Sigma$.

We have an obvious exact sequence

$$
1 \rightarrow B_{\infty} \rightarrow \mathcal{M}\left(E D^{*}\right) \rightarrow \mathcal{M}(E D) \rightarrow 1
$$

Using the embedding $\iota_{K}: T \hookrightarrow \mathcal{M}(E D)$ we can restrict $\mathcal{M}\left(E D^{*}\right)$ at $\iota_{K}(T)$. This restriction is the group $\mathcal{A}_{T, K}$, which fits also in the exact sequence

$$
1 \rightarrow B_{\infty} \rightarrow \mathcal{A}_{T, K} \rightarrow T \rightarrow 1
$$

The main example of a nontrivial cocycle is the one associated to the discrete Godbillon-Vey class. Specifically, identify $T$ with the group of dyadic piecewise affine homeomorphisms of $[0,1] / 0 \sim 1$. For any $\gamma \in T$ and $v \in \frac{\mathbb{Z}\left[\frac{1}{2}\right]}{\mathbb{Z}}$ we set

$$
K(\gamma, v)=\gamma^{\prime \prime}(v)=\log _{2} \gamma_{r}^{\prime}(v)-\log _{2} \gamma_{l}^{\prime}(v)
$$

where $\gamma_{r}^{\prime}, \gamma_{l}^{\prime}$ are the right respectively left derivatives of $\gamma$. It is well-known $\left.(25,27]\right)$ that $K(\gamma, v)$ is a cocycle.

The extension $\mathcal{A}_{T, K}$ obtained when $K$ is the Godbillon-Vey cocycle above is simply denoted $\mathcal{A}_{T}$. 
Remark 4.2. The definition from [29] was slightly different because it used $n+1$ instead of $n$ in the definition and we have punctures at the vertices of $\mathcal{T}$; in particular the vertex $v$ of $T$ was always sent into $\gamma(v)$. Nevertheless, the two groups $\mathcal{A}_{T}$ in [29] and the present paper coincide. Notice that there is a homeomorphism between the two differently punctured surfaces which slide all the punctures of the pending lines one unit such that their first punctures belong are now the vertices of $T$. This homeomorphism conjugates between the two versions of $\mathcal{A}_{T}$. Our version has the advantage of simplifying the already cumbersome computations of the next section.

\subsection{The abelianized extension $\mathcal{A}_{T}^{\mathrm{ab}}$}

Proposition 4.3. The class $c_{\mathcal{A}_{T}^{\text {ab }}} \in H^{2}(T)$ is given by $c_{\mathcal{A}_{T}^{\text {ab }}}=\alpha$.

Proof. This is already stated in [27]. In fact $\mathcal{A}_{T}$ splits over the cyclic subgroups $\mathbb{Z} / 2^{m} \mathbb{Z} \subset T$ for all $m$ and this implies that the coefficient of $\chi$ vanishes. Moreover, the coefficient of $\alpha$ is shown in [27] to be one.

Proposition 4.4. There is an isomorphism of extensions between $\mathcal{A}_{T}^{\text {ab }}$ and $T_{30,16,3,1}$.

Proof. We have to consider $\alpha$ and $\beta$ as elements of the group of homeomorphisms preserving the dyadics of $S^{1}$. Recall that $T$ has the standard generators $A, B, C$ from [10, as described in section 2.1. Realizing $A, B, C$ as (stable) couples of binary trees we can identify

$$
\beta=C^{-1}, \alpha=C^{-1} B
$$

and thus

$$
\beta(x)=\left\{\begin{array}{lll}
\frac{x}{2}+\frac{1}{2}, & \text { if } \quad x \in\left[0, \frac{1}{2}\right] \\
x+\frac{1}{4}, & \text { if } \quad x \in\left[\frac{1}{2}, \frac{3}{4}\right] \\
2 x-\frac{3}{2}, & \text { if } \quad x \in\left[\frac{3}{4}, 1\right]
\end{array} \quad \alpha(x)=\left\{\begin{array}{lll}
\frac{x}{2}+\frac{1}{2}, & \text { if } & x \in\left[0, \frac{3}{4}\right] \\
x+\frac{1}{8}, & \text { if } & x \in\left[\frac{3}{4}, \frac{7}{8}\right] \\
4 x-\frac{7}{2}, & \text { if } & x \in\left[\frac{7}{8}, 1\right]
\end{array}\right.\right.
$$

This implies that

$$
\beta^{\prime \prime}(0)=-2, \beta^{\prime \prime}\left(\frac{1}{2}\right)=1, \beta^{\prime \prime}\left(\frac{3}{4}\right)=1, \alpha^{\prime \prime}(0)=-3, \alpha^{\prime \prime}\left(\frac{1}{2}\right)=0, \alpha^{\prime \prime}\left(\frac{3}{4}\right)=1, \alpha^{\prime \prime}\left(\frac{7}{8}\right)=2
$$

In the enhanced rooted tree model we can therefore explain the action of $\alpha$ and $\beta$ as in the pictures below.

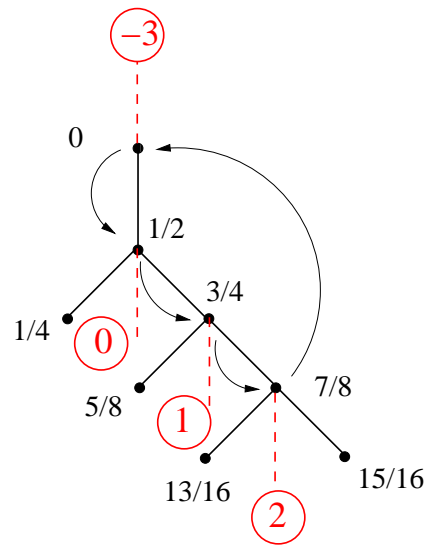

$\alpha$

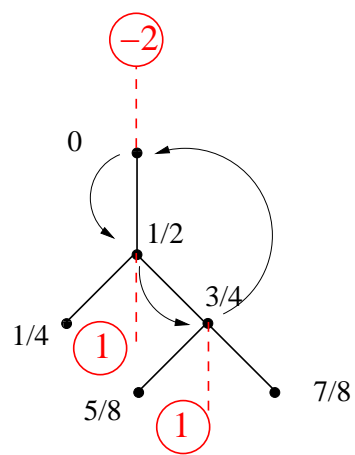

$\beta$

It follows by direct calculation that the action of $\beta \alpha$ is described by: 


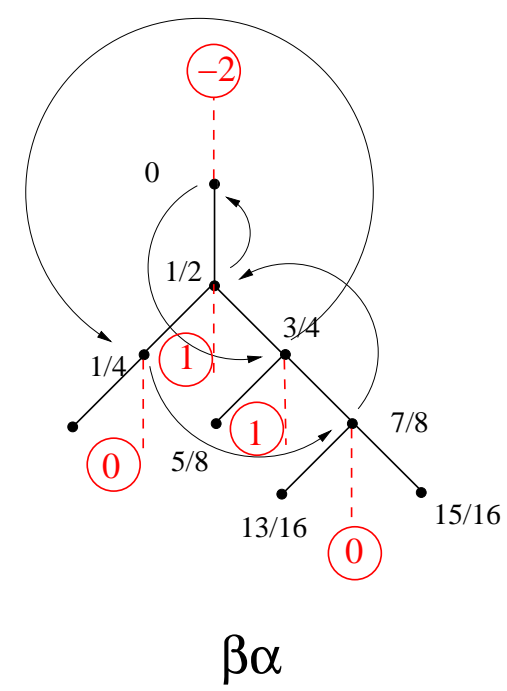

The problem we face now is to consider lifts $\alpha^{* *}$ and $\beta^{* *}$ of $\alpha$ and $\beta$ as mapping classes of homeomorphisms of the enhanced surface $E D^{*}$.

For the sake of simplicity we change in the pictures below the labels corresponding to the vertices of the rooted tree, as follows: $A$ states for $0, B$ for $1 / 2, C$ for $3 / 4, D$ for $7 / 8$ and $E$ for $1 / 4$. Moreover the pending line at $A$ has its vertices labeled $A_{1}, A_{2}, \ldots$ and so on for all other vertices.

The supports of these classes homeomorphisms correspond to suitable disks around the vertices.

1. $\beta^{* *}$ has a support a disk embedded into $E D^{*}$ containing $A_{i}, B_{i}, C_{i}$ for $i \leq 3$. Moreover the action of $\beta$ is described as follows: first $\beta$ acts as a rotation of order 3 in the plane; next the vertices $A_{1}$ and $A_{2}$ are slid in counterclockwise direction towards the positions $C_{1}$ and $B_{1}$, respectively. In meantime the punctures $C_{j}$ (and $B_{j}$ ) are simultaneously translated one unit along their pending lines and hence $C_{j}$ (respectively $B_{j}$ ) will arrive to the position formerly occupied by $C_{j+1}$ (respectively $B_{j+1}$ ), for all $j \geq 1$. The punctures $A_{j}$ (for $j \geq 3$ ) are translated simultaneously two negative units along their pending line and hence $A_{j}$ will arrive to the position formerly occupied by $A_{j-2}$.

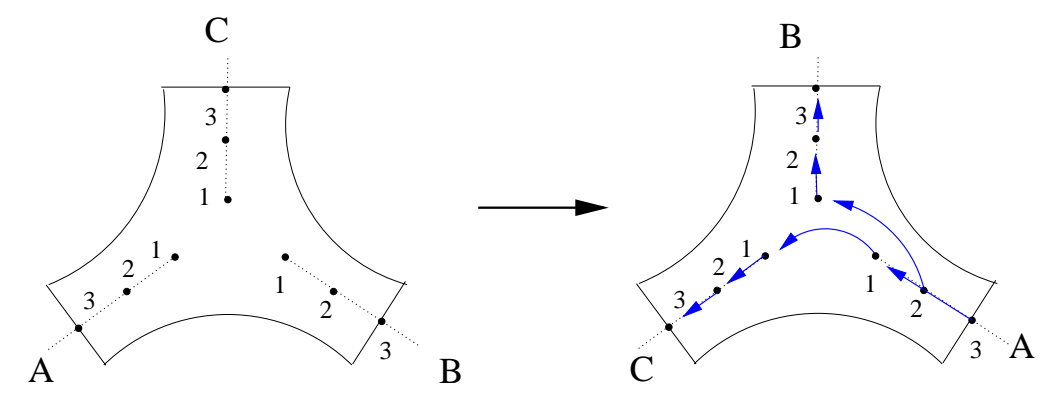

2. $\alpha^{* *}$ has a support a disk embedded into $E D^{*}$ containing $A_{i}, B_{i}, C_{i}, D_{i}$ for $i \leq 4$. The action of $\alpha$ is described as follows: first $\alpha$ acts as a rotation of order 4 in the plane; further $A_{1}$ and $A_{2}$ are slid in counterclockwise direction onto $D_{1}$ and respectively $D_{2}$, while $A_{3}$ is slid into $C_{1}$. The slidings occur simultaneously with the the translations of all punctures $D_{j}(j \geq 1)$ two units along their pending lines and the $C_{j}(j \geq 1)$ one unit along their pending line. Moreover the $A_{j}(j \geq 4)$ are translated three negative units along their pending line. The trajectories of the points are represented below (we did not figure the obvious translations along the pending lines): 


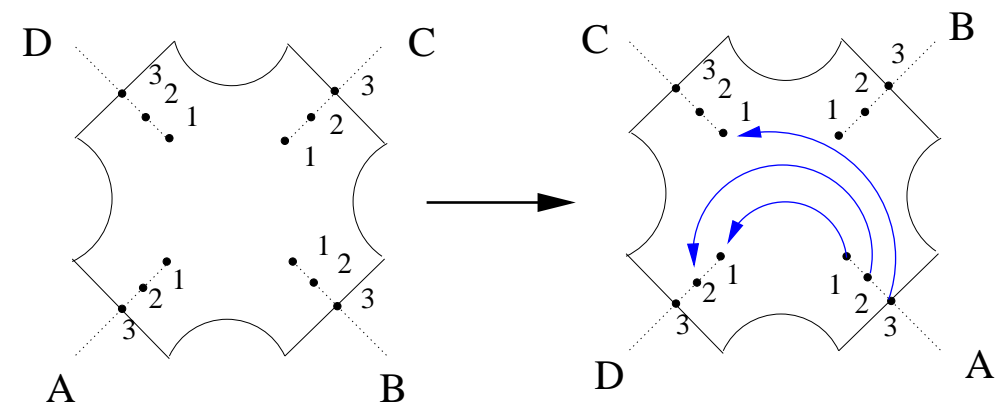

We are able now to figure out the element $\beta^{* *} \alpha^{* *} \in \mathcal{A}_{T}$. Its support is now a disk embedded into $E D^{*}$ containing $A_{i}, B_{i}, C_{i}, D_{i}, E_{i}$ for $i \leq 4$.

Notice however that the shape of the punctures trajectories is not sufficient for recovering the relative position of punctures. We have to specify somewhat the speed of each puncture along its trajectory, or equivalently, to specify a parameterization. There is a way to give a discrete parameterization by associating natural numbers to arcs of trajectories as follows. The time interval is divided into $N$ smaller intervals for some $N$. The arc $\lambda$ is given the label $k \in\{1,2, \ldots, N\}$ if the respective puncture travels along $\lambda$ precisely in the $k$-th interval of time. Actually this says that whenever we have two arcs (disjoint or not) labeled $j$ and $k$, with $j<k$ the respective punctures travel first along the arc $j$ and next along the arc $k$. A mapping class group element written as a word in the $\alpha^{* *}$ and $\beta^{* *}$ will lead naturally to a discrete parameterization. Moreover, $N$ is chosen such that the mapping class of the respective homeomorphism is uniquely determined by the discrete parameterization.

As an example $\beta^{* *} \alpha^{* *}$ is completely described by the following picture (including parameterization):
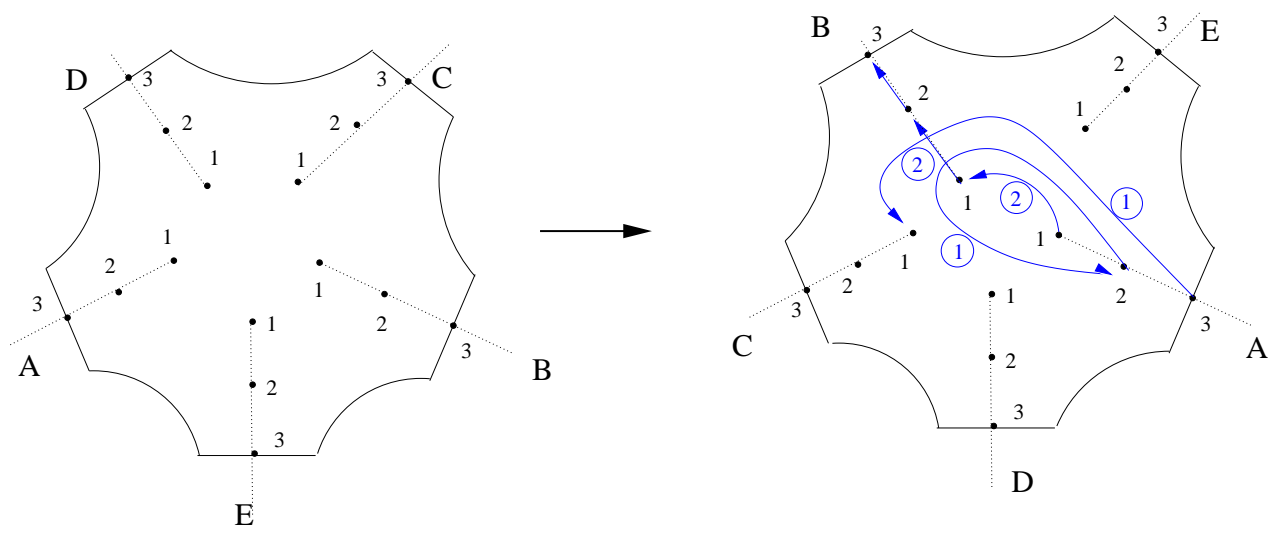

Recall that lifts in $\mathcal{A}_{T}$ of the relations in $T$ should give elements of the infinite braid group $B_{\infty}$, the kernel of the projection $\mathcal{A}_{T} \rightarrow T$. Denote by $a: \mathcal{A}_{T} \rightarrow \mathcal{A}_{T}^{\text {ab }}$ the projection homomorphism. The restriction $\left.a\right|_{B_{\infty}}: B_{\infty} \rightarrow Z$ is then identified to the abelianization homomorphism. We have therefore to compute the integers $n, p, q$ so that $a\left(\beta^{* * 3}\right)=z^{q}, a\left(\alpha^{* * 4}\right)=z^{p}, a\left(\left(\beta^{* *} \alpha^{* *}\right)^{5}\right)=z^{n}$.

Lemma 4.2. The braid $\beta^{* * 3} \in B_{\infty}$ is given by the picture below

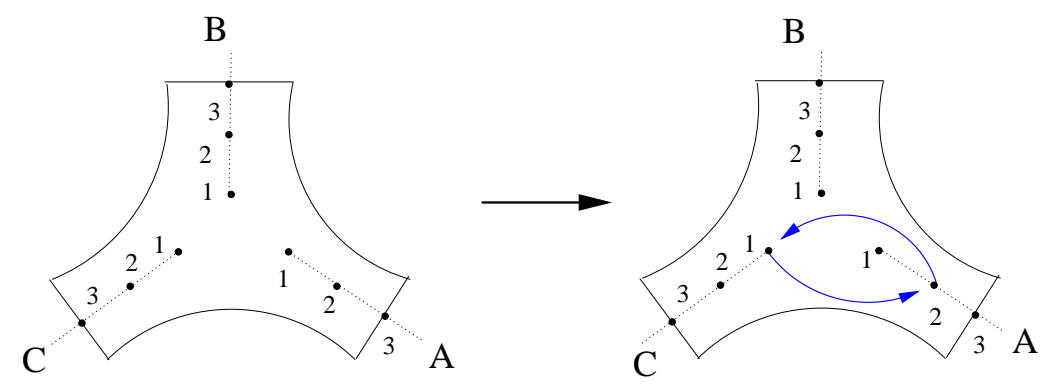


In particular $a\left(\beta^{* * 3}\right)=z^{3}$.

Proof. We will give an explicit proof of what is going on in this (simplest) situation. The terminology is somewhat unconventional. We say that punctures "travel" from one location to another, in certain intervals of time. One chooses the time intervals so that their simultaneous trajectories do not intersect and one can recover the class of the associated homeomorphism. It is of course sufficient to have a finite number of such intervals, which we also call steps, and the relative speed within each interval is not important, because any two speed values lead to isotopic homeomorphisms. Eventually we can compose the classes obtained by describing them step by step. The labels are assigned to the punctures and thus they travel around; at each step there are induced (infinite) permutations of the labels. At the end we find an element of a finite braid group inside $B_{\infty}$ and trajectories of punctures are now viewed as strands of that braid. Strands are said trivial if they are trivial as braid strands. We will exemplify below with $\beta^{* * 3}$.

Each time we have an action of $\beta$ there is a first step comprising an order 3 rotation in the plane and a second step, the sliding, where two punctures keep traveling in the counterclockwise direction, while the others are fixed, along a circle arc of angle $\frac{4 \pi}{3}$ (a $\frac{2}{3}$-turn) and respectively $\frac{2 \pi}{3}$, according to the picture of $\beta^{* *}$ : the puncture labeled $A_{1}$ is the one which travel faster while $A_{2}$ travels a shorter amount. There are also some translations along the pending lines so that, for instance, $B_{1}$ is sent into $B_{2}$ and $C_{1}$ onto $C_{2}$. In general, we will not bother to represent on the picture these translations, except when their final action is nontrivial.

Assume now that we want to compose two such classes $\beta^{* *}$. Then, we draw first the result of the first $\beta^{* *}$ action and further we resume with the first step, namely an order 3 rotation. One applies next the second step sliding and observe that the puncture labeled $A_{1}$ is again located in the position from which it has to be slid. Its fellow traveler is this time the puncture labeled $C_{1}$, which has moved at the previous step from position $C_{1}$ onto $C_{2}$.

It is clear now how to add one more $\beta^{* *}$ : an order 3 rotation sends the puncture labeled $A_{1}$ again into the position to be slid and thus it completes one more $\frac{2}{3}$-turn to arrive into its initial position. Its fellow traveler in the sliding step is the puncture labeled $A_{2}$, which followed before the path from $A_{2}$ to $B_{1}$ and then from $B_{1}$ onto the location of $B_{2}$. The sliding will send it into the location of $C_{1}$.

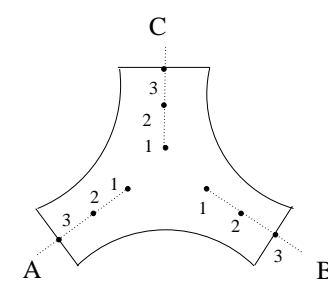

B

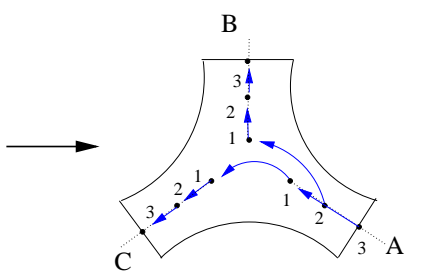

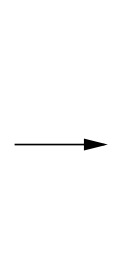
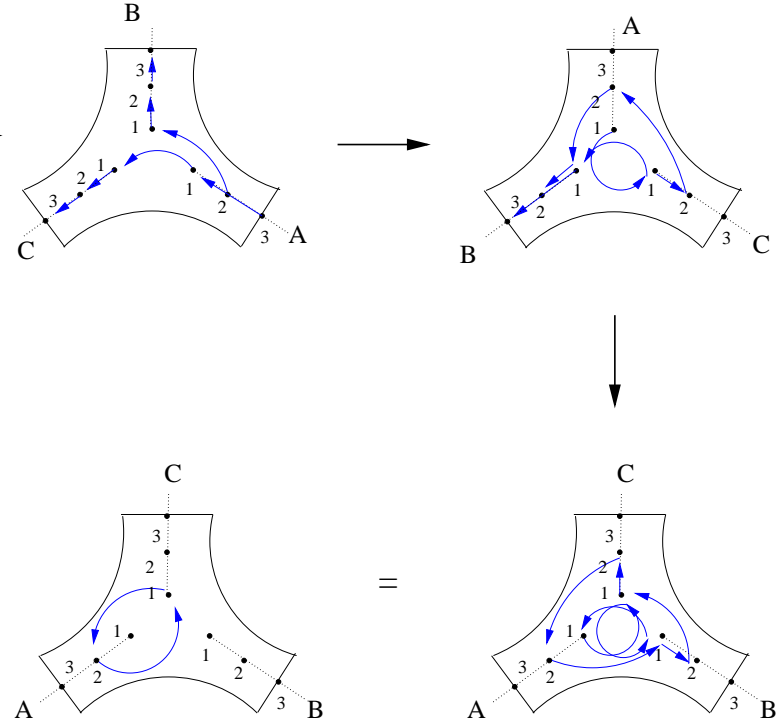

The trajectory of $A_{1}$ is a strand of the braid $\beta^{* * 3}$ which can be split off. This implies that, by means of an isotopy we can assume that $A_{1}$ is fixed. This isotopy corresponds to shrinking the trajectory of $A_{1}$ to a point. This shrinking could be done without touching the others trajectories, which means that it lifts to an isotopy between braids in the three dimensional space. It follows that the only nontrivial part of the braid $\beta^{* * 3}$ is the exchange between the punctures $A_{2}$ and $C_{1}$. The translations along the pending lines yield trivial strands for the remaining punctures. 
The remaining calculations are of the same sort, but involve more complicated braids. We were unable to find the braids (as the two dimensional picture is misleading) but we will make use of additional simplifications to help computing the images under the abelianization map.

First, one can find $a(\sigma)$ using only the winding numbers of the trajectories of the braid $\sigma$. Let $\sigma$ be given as a geometrical braid in $\mathbb{R}^{2} \times[0,1]$ by the parameterizations $\left(x_{k}(t), t\right)$, for $t \in[0,1]$, each subscript $k$ corresponding to one strand. The (relative) winding number $\nu(j, k)$ of the strands $j$ and $k$ is the angle that the vector $x_{j}(t)-x_{k}(t)$ swept when $t$ goes from 0 to 1 . Turning counterclockwise yields positive angles. If the punctures sit all on the same line in $\mathbb{R}^{2} \times 0$ then relative winding numbers are multiples of $\pi$. However, they make sense even when the punctures are given arbitrary positions in the plane. The (total) winding number $\nu(\sigma)$ is the sum of all relative winding numbers of its (distinct) strands.

Lemma 4.3. We have $\pi a(\sigma)=\nu(\sigma)$.

Proof. Both sides are group homomorphisms and their values on the generators coincide.

It is much simpler to compute winding numbers when trajectories are known.

Lemma 4.4. We have $\nu\left(\alpha^{* * 4}\right)=16 \pi$.

Proof. We draw each trajectory individually, along with its discrete parameterization. The only nontrivial trajectories are those of the strands starting at $A_{1}, A_{2}, A_{3}$ and $D_{1}$. The remaining ones are easily shown to be trivial and having zero winding numbers with all others.

$\mathrm{C}$

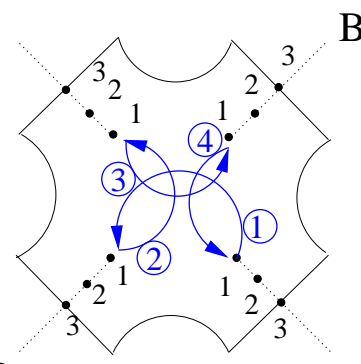

$\mathrm{D}$
B

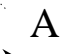

C

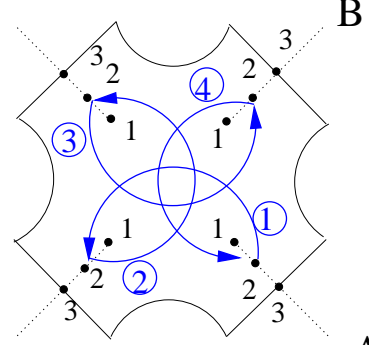

D

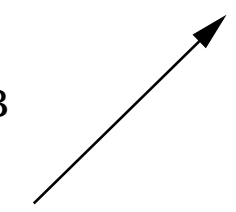

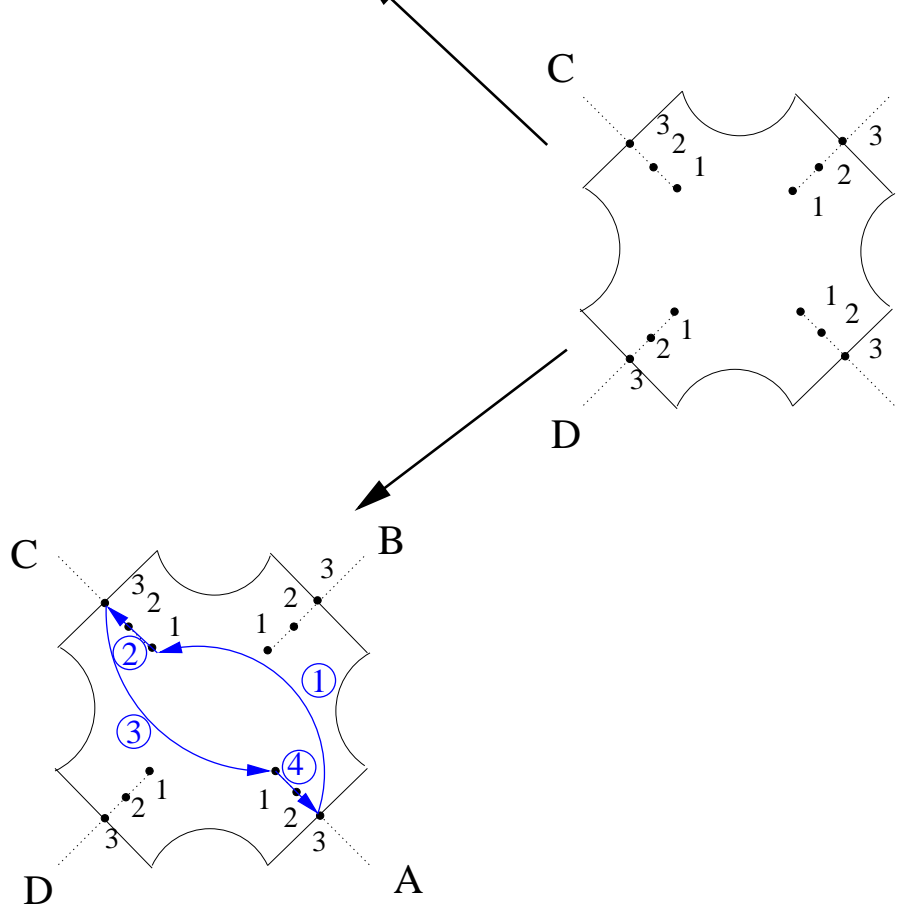

A
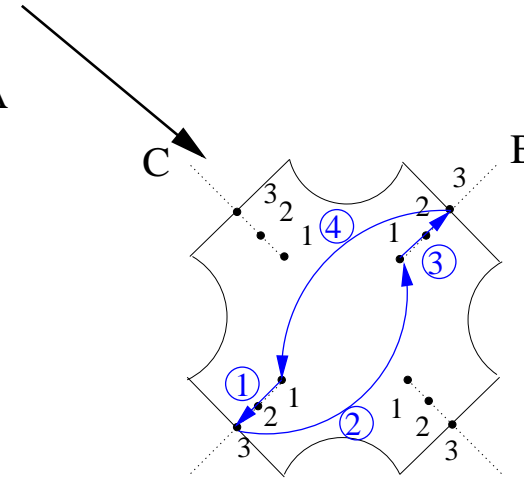

D 
Then we compute easily from the picture above the relative winding numbers between strands (hereby identified to their start-point):

$$
\nu\left(A_{1}, A_{2}\right)=6 \pi, \nu\left(A_{1}, A_{3}\right)=\nu\left(A_{2}, A_{3}\right)=\nu\left(A_{3}, D_{1}\right)=\nu\left(A_{2}, D_{1}\right)=\nu\left(A_{1}, D_{1}\right)=2 \pi
$$

This ends the proof of the Lemma.

Lemma 4.5. We have $\nu\left(\left(\beta^{* *} \alpha^{* *}\right)^{5}\right)=30 \pi$.

Proof. Recall that pictures define mapping classes of homeomorphisms. Then, for any mapping classes $H_{1}$ and $H_{2}$ for which the compositions below make sense and represent elements of $B_{\infty}$ we have the identity

$$
a\left(H_{1} \circ \rightleftharpoons \circ \circ H_{2}\right)-a\left(H_{1} \circ \leftrightarrow \cdot \cdots H_{2}\right)=2
$$

In fact the first braid is obtained from the second one by inserting some $\sigma^{2}$, which braids two punctures twice. Replacing the first braid by the second one will be called a (direct) simplification of the braid diagram.

We can use a direct simplification within the picture of $\beta^{* *} \alpha^{* *}$ in order to remove the loop trajectory based at $A_{2}$. Thus after 5 simplifications within $\left(\beta^{* *} \alpha^{* *}\right)^{5}$ we obtain the following trajectories.

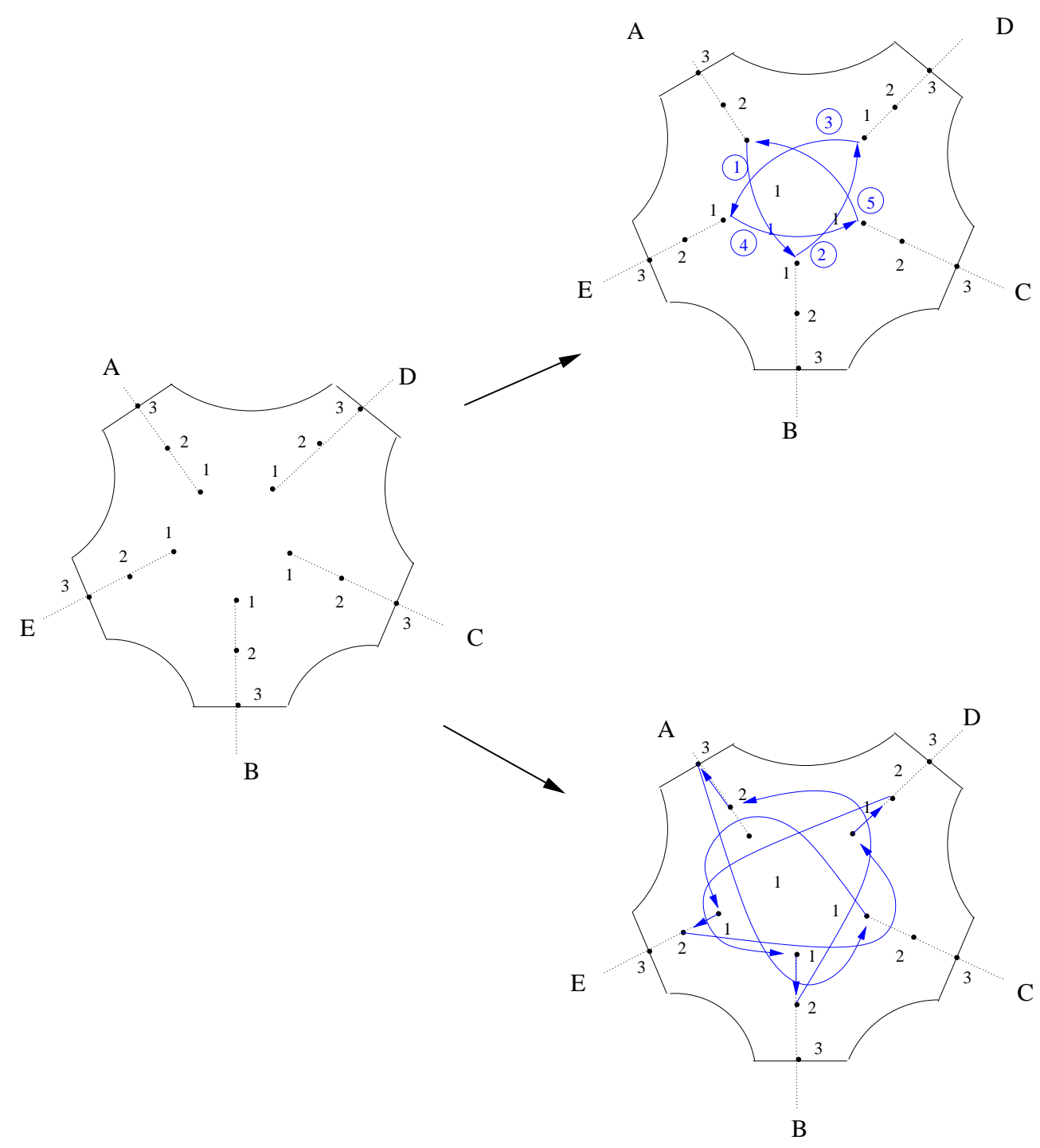


We did not draw but the nontrivial trajectories which are of two types: a long trajectory of the strand starting at $A_{1}$ and a braid cycle permuting circularly the nine punctures $A_{2}, A_{3}, C_{1}, E_{1}, E_{2}, D_{1}, D_{2}, B_{1}$ and $B_{2}$. The braid cycle admits 4 direct simplifications by sliding $C_{1}$ outward to $E_{2} D_{1}, B_{1}$ outward $A_{3} C_{1}, E_{1}$ outward of $D_{2} B_{1}$ and $D_{1}$ outward of $B_{2} A_{2}$ and becomes the following braid $b$ pictured below:

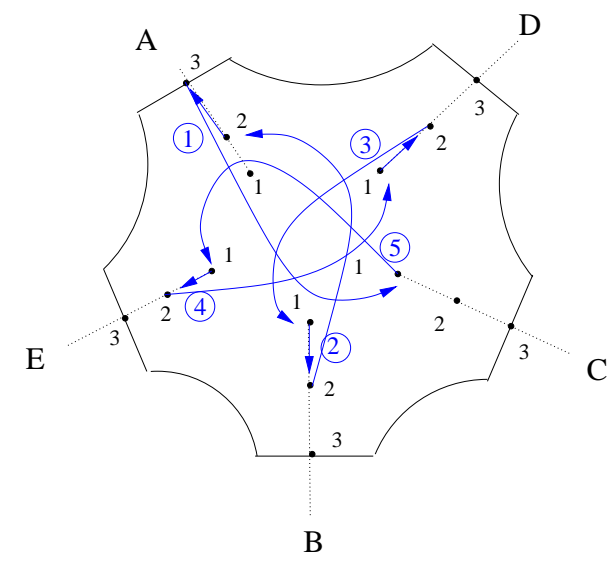

By direct calculation (taking care of the discrete parameterizations for both $b$ and the trajectory of $A_{1}$ ) we obtain

$$
\sum_{X} \nu\left(A_{1}, X\right)=4 \pi
$$

where the sum is over all strands $X$ of the braid $b$. Since the braid $b$ is now a cycle of length 9 we find that $\nu(b)=8$. Summing up all contributions to $\nu\left(\left(\beta^{* *} \alpha^{* *}\right)^{5}\right)$ we obtain 30 , as claimed.

Remark 4.3. The elements $\beta, \alpha, \beta \alpha$ are respectively of order 3,4 and 5 . Since the Euler class of $\mathcal{A}_{T}$ is zero, it splits over cyclic subgroups of $T$ and the same holds then for $\mathcal{A}_{T}^{\text {ab }}$. This implies that $q \equiv 0(\bmod 3)$, $p \equiv 0(\bmod 4), n \equiv 0(\bmod 5)$. Moreover, the permutation induced by $\left(\beta^{* *} \alpha^{* *}\right)^{5}$ is a cycle of length 9 and thus is even. In particular $n \equiv 0(\bmod 2)$ and so $n \equiv 0(\bmod 10)$.

\subsection{End of the proof of Theorem 1.2}

In order to finish the proof we need to compute $\chi(n, p, q, r)$. We have first:

Proposition 4.5. We have $\chi(n, p, q, r)=12 n-15 p-20 q+c r$, for some $c \in \mathbb{Z}$.

Proof. It suffices to use Proposition 2.2 and the linearity of the function $\chi(n, p, q, r): \mathbb{Z}^{4} \rightarrow \mathbb{Z}$. For the later, use the arguments from the proof of lemma 2.2 where the case $r=0$ is treated. We skip the details.

Propositions 4.3 and 4.4 show that $\chi(30,16,3,1)=0$, and hence $\chi(n, p, q)=12 n-15 p-20 q-60 r$. Then proposition 4.2 finishes the proof of theorem 1.2

The analysis above can be used in establishing also the following:

Proposition 4.6. The group $\mathcal{A}_{T, K}$ is finitely generated.

Proof. Consider the group $\mathcal{A}_{T}$, the general case following the same way. The elements $\alpha^{* *}$ and $\beta^{* *}$ generate the quotient $T$ and it suffices to add sufficiently many elements to be able to generate all of $B_{\infty}$, which is the braid group on the punctures. Let $\sigma$ be the braiding of the first two punctures of the pending line at $A$. Let denote by $t$ the mapping class of the homeomorphism which translates one unit along the line made by gluing together the two (half)-lines pending at $A$ and $B$. Observe now that $t$ conjugate $\sigma$ to any of the braid generators of the pending line at $A$ and $B$. On the other hand the action of $T$ by conjugation on $\mathcal{A}_{T}$ is transitive on the set of pending lines, as it is the action of $P S L(2, \mathbb{Z})$ on the binary tree. This means that for vertex of the rooted binary tree there exists a word $w=w\left(\alpha^{* *}, \beta^{* *}\right)$ such that the corresponding element of $\mathcal{A}_{T}$ sends the pending line at $v$ asymptotically onto the pending line at $A$. The meaning of the word asymptotically is that all but finitely many vertices of the respective line are sent into the other ones. The 
first few vertices might be sent onto other pending lines, as it happens with $\alpha^{* *}$ and $\beta^{* *}$ which slide finitely many points. However sliding of punctures occur only at the supports of $\alpha^{* *}$ and $\beta^{* *}$. Thus the line pending at $v$ is sent into the line pending at $A$ and slidings of its vertices could appear only when its first vertex will reach the set $\left\{A_{1}, B_{1}, C_{1}, D_{1}, E_{1}\right\}$. Let then $M$ be the mapping class group of an admissible subsurface containing the supports of $\alpha^{* *}$ and $\beta^{* *}$. It follows that adjoining the generators of $M$ to $\alpha^{* *}, \beta^{* *}, \sigma, t$ we generate all of $B_{\infty}$ and thus $\mathcal{A}_{T}$.

\section{Odds and ends}

\subsection{Geometric extensions}

We would like to understand all extensions

$$
1 \rightarrow B_{\infty} \rightarrow G \rightarrow T \rightarrow 1
$$

coming out from nature. A tentative approach is to say that such an extension is geometric if there exists a tessellation of a planar surface $\Sigma$ with infinitely many punctures such that $G$ is the asymptotic mapping class group of $\Sigma$ with this extra structure (see also [22]). Then $B_{\infty}$ is the braid group in the punctures.

In order to avoid trivial constructions we restrict to those examples which are minimal in some sense. The simplest minimality condition is to ask the natural homomorphism $T \rightarrow \operatorname{Out}\left(B_{\infty}\right)$ to have one orbit of generators of $B_{\infty}$ i.e. that $T$ acts transitively on the first homology of the surface $\Sigma$. Alternatively, this amounts to require that the lifts of the generators $\alpha$ and $\beta$ of $T$ together with a standard braid generator $\sigma$ form a generator system for $G$.

The groups $T_{1,0,0}$ and $T_{3,1,0}$ are geometric (see 23]) and minimal. Although $\mathcal{A}$ is also geometric one needs to modify the minimality condition above in order to be fit for it.

It seems that there are only finitely many such minimal geometric extensions, for appropriate minimality conditions.

\subsection{Finite surfaces}

It is known that the mapping class group $\mathcal{M}(\Sigma)$ of a punctured surface $\Sigma$ embeds into the groupoid of flips acting on the triangulations of $\Sigma$ with vertices at punctures. Quantization of the Teichmüller space of the surface $\Sigma$ lead then by the technology of [19] to projective representations of the mapping class group $\mathcal{M}(\Sigma)$ and thus to a central extension $\widehat{\mathcal{M}(\Sigma)}$.

Recall also that $H^{2}(\mathcal{M}(\Sigma))$ is freely generated by the Euler class $\chi$ together with the classes corresponding to each one of the punctures.

It seems plausible that the class $c_{\widehat{\mathcal{M}(\Sigma)}}$ of the extension is actually equal to $12 \chi \in H^{2}(\mathcal{M}(\Sigma))$.

Notice that additional work is needed for obtaining this result because of our lack of knowledge of the Ptolemy groupoids and their associated groups (see [32]) for finite surfaces of positive genus.

\section{References}

[1] E.W.Barnes, The genesis of the double gamma function, Proc. London Math. Soc. 31(1899), 358-381.

[2] S.Baseilhac and R.Benedetti, Classical and quantum dilogarithmic invariants of flat PSL(2,C)-bundles over 3manifolds, Geometry \& Topology 9(2005), 493-569.

[3] Hua Bai, F.Bonahon and Xiaobo Liu, Local representations of the quantum Teichmüller space, math.GT/0707.2151.

[4] R.Baxter, Exactly solvable modles in statistical mechanics, Academic Press, 1982.

[5] F.Bonahon and Xiaobo Liu, Representations of the quantum Teichmüller space and invariants of surfaces diffeomorphisms, Geometry \& Topology 11(2007), 889-937.

[6] M.G.Brin, The chameleon groups of Richard J. Thompson: automorphisms and dynamics, Inst. Hautes Études Sci. Publ. Math. No. 84, 1996, 5-33. 
[7] M.G.Brin, The Algebra of Strand Splitting. I. A Braided Version of Thompson's Group V, J. Group Theory 10 (2007), no. 6, 757-788.

[8] M.G.Brin, The Algebra of Strand Splitting.II. A Presentation for the Braid Group on One Strand, Internat. J. Algebra and Comput. 16(2006), 203-219.

[9] D. Calegari, Circular groups, Planar groups and the Euler class, Proceedings of the Casson Fest, Geom. Topol. Monogr. 7(2004), 431-491.

[10] J.W.Cannon, W.J. Floyd, and W.R. Parry, Introductory notes on Richard Thompson's groups, Enseign. Math. 42(1996), 215-256.

[11] L.Chekov and V.Fock, Quantum Teichmüller space, math.QA/9908165

[12] P.Dehornoy, Geometric presentations for Thompson's groups, Journal Pure Appl. Algebra, 203(2005), 1-44.

[13] P.Dehornoy, The group of parenthesized braids, Advances Math. 205(2006), 354-409.

[14] L.D.Faddeev, Discrete Heisenberg-Weyl group and modular group, Lett. Math. Phys. 34(1995), $249-254$.

[15] L.Faddeev and R.Kashaev, Quantum dilogarithm, Mod. Phys. Lett. A 9(1994), 427-434.

[16] V.Fock, Dual Teichmüller spaces, math dg-ga/9702018.

[17] V.Fock and A.B.Goncharov, Moduli spaces of local systems and higher Teichmüller theory, Inst. Hautes Études Sci. Publ. Math. no.103, 2006, 1-211.

[18] V.Fock and A.B.Goncharov, Moduli spaces of convex projective structures on surfaces, Advances Math. 208(2007), 249-273.

[19] V.Fock and A.B.Goncharov, The quantum dilogarithm and unitary representations of cluster modular groupoids, Invent. Math. 175(2009), 223-286.

[20] V.Fock and A.B.Goncharov, Cluster ensembles, quantization and the dilogarithm II: The intertwinner, arXiv:math/0702398

[21] L.Funar, Ptolemy groupoids actions on Teichmuller spaces, Modern Trends in Geometry and Topology, Deva, Romania 2005, (D.Andrica, P.Blaga, S.Moroianu, Eds.), Cluj Univ.Press 2006, p.185-201.

[22] L.Funar, Braided Houghton groups as mapping class groups, Annales Sci. Univ. "A.I.Cuza" Jassy, special vol. to the memory of Gh.Ionesei, 53(2007), 229-240.

[23] L.Funar and C.Kapoudjian, The braided Ptolemy-Thompson group is finitely presented, Geometry \& Topology 12(2008), 375-430.

[24] L.Funar and C.Kapoudjian, The Ptolemy-Thompson group $T^{*}$ is asynchronously combable, math.GT/0602490.

[25] E.Ghys and V.Sergiescu, Sur un groupe remarquable de difféomorphismes du cercle, Comment.Math.Helv. 62(1987), 185-239.

[26] A.B.Goncharov, Pentagon relation for the quantum dilogarithm and quantized $\mathcal{M}_{0,5}^{\mathrm{cyc}}$, Geometry and dynamics of groups and spaces, In memory of Alexander Reznikov (M.Kapranov, S.Kolyada, Yu.I.Manin, P.Moree and L.Potyagailo Ed.), 415-428, Progr. Math., 265, Birkhäuser, Basel, 2008.

[27] P.Greenberg and V.Sergiescu, An acyclic extension of the braid group, Comment. Math. Helv. 66(1991), $109-138$.

[28] R.Kashaev, Quantization of Teichmüller spaces and quantum dilogarithm, Lett. Math. Phys. 43(1998), 105-115.

[29] C.Kapoudjian and V.Sergiescu, An extension of the Burau representation to a mapping class group associated to Thompson's group T, Geometry and dynamics, 141-164, Contemp. Math., 389, Amer. Math. Soc., Providence, RI, 2005.

[30] P. Lochak and L. Schneps, The universal Ptolemy-Teichmüller groupoid, in Geometric Galois actions, vol. 2, L.M.S. Lecture Notes Ser., 243, Cambridge Univ.Press, 1997.

[31] R.C.Penner, Universal constructions in Teichmuller theory, Advances Math. 98(1993), 143-215.

[32] R.C.Penner, The universal Ptolemy group and its completions, Geometric Galois actions, 2, 293-312, L.M.S. Lecture Notes Ser., 243, Cambridge Univ. Press, Cambridge, 1997. 
